\title{
Emergence and Evolution of Meaning
}

\author{
José M. Díaz Nafría" and Rainer E. Zimmermann"* \\ "Universidad de León, Spain; Hochschule München, Munich, Germany, diaz-naf@hm.edu \\ "Fakultät 13, Hochschule München, Munich, Germany, rainer.zimmermann@hm.edu
}

\begin{abstract}
The category of meaning is first traced forwards starting from the origin of the Universe itself and its grounding in pre-geometry; then it is traced backwards from the sense-interactions within the world to the interpretation of the corresponding reality. Different from many former approaches in the theories of information and also in biosemiotics in our progressive perspective, we show: on the one hand, that the forms of meaning emerge alongside with information and energy; on the other, that information can be visualised as being always meaningful - in a sense to be clarified, which extends Floridi's General Definition of Information - rather than meaning showing up as a later specification of information within social systems only. In the regressive perspective the category of meaning is explored starting from the manifestation of reality in its own level of interaction. Based upon the physical constraints of the manifestation through electromagnetic waves generated by an object of observation, which constitutes the basis of animal vision, we analyse the limits of the meaning-offer of such manifestation. This allows us: (1) to compare the efficiency of natural evolution in the reception of such meaning-offer; (2) to analyse the conditions for developing a hermeneutical agency able to acknowledge the reality underlying its manifestation. Hence, what we actually do - through this dual perspective - is to follow the strict line of the Unified Theory of Information in the sense of Hofkirchner, visualising information and energy as two different categorical aspects of one and the same underlying primordial structure.
\end{abstract}

Keywords: Philosophy of Information, Unified Theory of Information, Meaning, Quantum Gravity, Electromagnetic Theory, Perception, Biosemiotics

\section{Introduction}

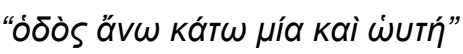

[The way up and the way down are one and the same]

(Heraclitus of Ephesus, DK 60)

From a dualist perspective, one could be the way down, in which reality is ordered, and other the way up, in which it is interpreted, i.e. in which it acquires meaning (for a subject). The classical problem attached to this view concerns the communication of substances, which can be rephrased as: if both ways are independent from each other, how could the starting point be reached? How can reality be properly referred to by the meanings possessed by a subject?

When speaking of information as something necessary meaningful - as it is for instance conceived by Floridi's General Definition of Information (GDI) - and disseminated all over the world as considered by the sciences, well beyond human contexts - the problem can be stated as: How Information acquires meaning in the first place and for the different contexts. Floridi addresses this issue in terms of the Symbol Grounding Problem in a way we have criticized elsewhere (Zimmermann and Díaz Nafría 2012), basically because by tackling the problem in its epistemic aspects, he is dragging-in the aforementioned hindrances of the dualist perspective. Instead, we prefer to hold with Heraclitus an intrinsic continuity between the way up and the way down, adopting instead an onto-epistemic stance, therefore rather monist, which can be very well represented by the Spinozist proposition "the order and the connection of ideas is the same as the order and the connection of things" (Spinoza 1677, II prop.7). Nevertheless, it is important to notice that our position neither implies the reversibility of the interpretation cycle, nor that the world is as we represent it. On the contrary, the interpretation cycle is in strict sense irreversible, similar to thermodynamic cycles. And such irreversibility is indeed essential to the evolution of complexity in the universe from the most elementary interaction of matter - as represented by spin networks - to the creation of molecules, biological structures, cognition and social systems.

This progressive perspective, aimed at understanding the emergence of meaning from pregeometry to reflexive meaning, is developed in section 2, based upon a common "skeleton-of-theuniverse". While arguing the grounds of this vision in $\S 2.1$, we intend to draw attention to the fact that - as happened with the conceptualization within $19^{\text {th }}$ century physics, which forged the grounds for a scientific understanding of information, chiefly manifested from the 1940s (Segal 
2003) - remarkable developments in fundamental physics for the last decades are currently fostering new insights into the understanding of information. As we will show, such insights are additionally connected to the endeavour of solving one of the most challenging problems for the unification of the sciences (namely the reconciliation of the theories of relativity and quanta), though such insights concerning information have not properly been acknowledged within the field of information studies. Thus its integration within the framework of developing a common understanding of information might provide robust roots for the formulation of a Unified Theory of Information (UTI) as proposed by Hofkirchner and others (Hofkirchner 1999; 2010). As discussed in §2.2, a generalized concept of autonomous agency underpins the proper development of the GDI throughout nature, as something which is always meaningful. To this end, meaning is related to an effective course of action, so, instead of being regarded as an epistemological category - like in Floridi - it is conceived onto-epistemologically. This general understanding of information and meaning enables the visualisation - as argued in $\$ 2.3$ - of information alongside energy, referring to the potentiality of selecting or producing changes respectively; and, correspondingly, structure alongside matter, as the actualization of the selected or realised changes respectively. Moreover this broad understanding provides a way for overcoming Capurro's trilema (Capurro et al. 1999, 9 sq.), which states the necessity of choosing among univocity, analogy or equivocity when speaking of information throughout different contexts - something that was indeed overcome in the nineteenth century with respect to energy.

In section 3, the "skeleton-of-the-universe" (previously suggested as the basis for the downwards path corresponding to the hierarchical evolution of complexity) serves as a foothold for an upwards pathway corresponding to the interpretation of reality. As analysed in this section, the fundamentality of the emergence of regularities and meaning, argued in the previous section, imposes essential constraints to the interaction within the world when we aim at interpreting it. One of these emergences - relevant to our means of awareness - is represented by electromagnetic fields, which correspond to the regularity arising from the interaction of a more elementary level of matter (§3.1.1). Our vision (even if assisted by microscopic techniques) is strictly constrained to the structural regularities of the electromagnetic fields (\$3.1.2). We will show from the corresponding structural constraints that the world is not as we observe it (§3.2). Instead, the manifestation of reality itself - no matter what sensing ability the autonomous agents possesses - contains a fundamental ambiguity that has to be somehow solved by the agent in order to enable a proper response according to the corresponding level of interaction. To this end, since hermeneutical agents are also products of the world (i.e., they are attached to the, so to speak, same rules of the game which chiefly concerns the effectiveness of the interaction), the very complexity of the mechanism of awareness at a high level of complexity (evolving from an objective to a reflexive response as analysed in §3.3) has the possibility of creatively imagining reality, similarly as the world creates it. Information - in a cognitive sense, which can be derived from a wider perspective of information in other natural processes - corresponds to the actualization of this creative imagination while interacting with the world. This interaction imposes a non-reversible path in the round trip of constituting reality (within which the reflexive observer has come into existence) and interpreting it (by the observer herself).

In the conclusive remark (\$4) we compare our approach with other frameworks advanced in the converging fields of information, computation, cognition and communication, showing that our scaffolding provides new grounds for the development of the Unified Theory of Information Programme, as well as the possibility for bringing among different endeavours to solve common challenges.

\section{The Progressive Perspective: Top-Down}

\subsection{Information in Fundamental Physics}

After roughly 35 years of development in the theories of self-organization and related variants (chaos, self-organized criticality and so forth), it is somewhat of a surprise that the insights from physics proper have not yet sufficiently been heard in the ongoing quest for a precise concept of information (Hofkirchner 1999; 2012; Floridi 2011; Díaz Nafría and Salto 2009). As Seth Lloyd points out in his book from 2006 (Lloyd 2006, 52), already as early as in the sixties of the last century Fredkin and Zuse visualized the universe as a digital computer. This is a line of argument that Wolfram has followed more recently in his work on cellular automata published in 2002, not to speak of the more recent theories on quantum information (Benenti et al. 2007, Berman et al. 1998) which generically couple to theories of quantum gravity. For these physical theories, information plays a key role in a sense clearly transcending its classical understanding, as patently 
expressed by David Deutsch (one of the leading protagonists in these fields): “... [b]its, Boolean variables, and classical computation are all emergent or approximate properties of qubits, manifested mainly when they undergo decoherence" (Deutsch 2004, 93). In other words: "The world is made of qubits ... What we perceive to some degree of approximation as a world of single-valued variables is actually part of a larger reality in which the full answer to a yes-no question is not just yes or no, nor even both yes and no in parallel, but a quantum-observable - something that can be represented as a large Hermitian matrix" (Ibid., 100).

This line of argument actually goes back as far as to John Wheeler in 1977 for whom "... [a] true observation of the physical world ... must not only produce an indelible record, [but] somehow in part meaningful information" (Davies 2004, 8 - our emphasis). For him, “... [m]easurement implies a transition from the realm of mindless material stuff to the realm of knowledge. So it [is] not enough ... that a measurement should record a bit of information, that lowly bit had to mean something" (Ibid. - our emphasis). This perspective led at the time to the famous "it-from-bit" thesis proposing that "the universe [is] fundamentally an information processing system from which the appearance of matter emerges at a higher level of reality" (Ibid., 10). In fact, it is Seth Lloyd himself who after all has developed the cosmological implications in most detail when presenting his work on the computational universe (Lloyd 2006; 2010). For him, the big bang was also a bit bang (Penrose 1994,96$)$.

Within the theories of quantum gravity, these aspects have gained even more pertinence. This is because the quantum viewpoint itself typically tends to conceptualize information (contrary to Haefner's assertion (1999, xv): "at the physical level, we encounter a set of physics theories that have never considered information as an appropriate term to understand physical phenomena"). As Carlo Rovelli has concluded: "... what precisely quantum mechanics is about is the information that physical systems have about one another" (Rovelli 2004, 19). The quantum aspect itself however, turns out to be somewhat more involved than expected, as Roger Penrose has pointed out in his more recent works when he talks, for example, of what he calls quanglement in demonstrating his reluctance to utilize the concept of quantum information. As he says: "Quantum is not information, but [it] can be used in conjunction with ordinary information channels, to enable these to achieve things that ordinary signaling alone cannot achieve" (Penrose 1994, 603, 607).

It is especially in loop quantum gravity that these features are most prominent. The idea is that "[j]ust as a polymer, although intrinsically 1-dimensional, exhibits 3-dimensional properties in sufficiently complex and densely packed configurations, the fundamental 1-dimensional excitation of geometry can be packed appropriately to provide a geometry which, when coarse-grained on scales much larger than the Planck length, resembles continuous geometries" (Ashtekar 1998, 181). The theory is named after the Wilson-type loops which are essentially closed curves carrying quantized electric flux and being organized into hexagonal networks called spin networks (Smolin 2000, 135; Smolin 2004, 504). To be more precise, the significant objects are not just the loops, but their holonomies: they represent a generalized kind of parallel transport that can be described in terms of a Lie group element in the fibre bundle attached to the chosen base manifold. Hence, holonomies can be visualized as homomorphisms from some group structure defined in terms of equivalence classes of closed curves onto a Lie group. We can see then that essentially, "the result of evaluating a Wilson loop about a very small planar circle around a point $\mathrm{x}$ is proportional to the area enclosed by this circle times the corresponding value of the curvature tensor of the gauge field evaluated at x" (L.Kauffman 1998, 79; cf. Baez 1994). Hence, the holonomy has the same information as the curvature at this point (cf. Gambini 1996, 1 sq.). A spin network then, is a linear combination of products of holonomies of closed curves that wrap along the graph (Rovelli 2004, 237). Louis Kauffman who dealt with a representation of loops and knots in terms of (mathematical) category theory, has shown that in principle, the binor identity characterizing spin networks, the skein identity of the bracket polynomial in knot theory, and the trace identity are really all the same. Hence, space altogether shows up then as one of the possible targets of the many functors that extract information from the network (L.Kauffman 1998, 277 sq.) (For general networks see e.g. Barabási (2002), for categories see in particular Lawvere and Rosebrugh (2003), for an alternative approach in terms of strings see Susskind and Lindesay (2005). As to the relationship between functors and knots see also Yetter (2001), and Zimmermann (2000; 2002))

As a preliminary conclusions of all of this we can note the following.

First, the physicist's quest for a unified theory from the outset (an enterprise in fact that already starts at the end of the $19^{\text {th }}$ century) justifies that the concept of information is always present in the sense that comparatively early it became necessary to map the physical processes involved by means of thermodynamical (and statistical) techniques. From the beginning therefore, for Penrose, the entropy of a state is described as a measure of the volume of that compartment which contains 
the phase-space point which actually represents this state (Penrose 1989, 313). Hence, if a theory of cosmology must, as Smolin puts forward (Smolin 1997, 291), in order to be self-consistent, be a theory of the self-organization of the universe, the very aspect of organization entails a concept of information on an equal footing with the concept of energy. (A point, in fact, Floridi would agree with $(2011,135)$. See also Jantsch (1982)). This idea became popular back in the late seventies of the last century following the international reception of the theories of René Thom and llya Prigogine (Thom 1973; 1975; 1983; Prigogine 1979; 1996; cf. Zimmermann 1974-1990). As one can clearly recognize from this development, the problem of organization is closely related to the problem of a unified theory of physical interactions. Although significant progress has been achieved here, starting with Maxwell's theory of electromagnetism and with Einstein's theories of relativity, leading forward to a further unification including weak interactions (Salam-Weinberg-Glashow) and even to a GUT (Grand Unified Theory), gravitation (completing a true TOE, Theory of Everything) has not yet been successfully integrated into this enterprise. And the reason for this may be a deficiency in the proper co-ordination of energy and information within the theories of the cosmological beginnings. Looking particularly for characteristic differences in the entropy of the universe, in 1979 , Roger Penrose has claimed a principle of time-asymmetry which shows up as a direct consequence of this evolution of entropy and can be formulated as an explicit energy condition (called the Weyl curvature hypothesis) (cf. Halliwell et al. 1994; Hawking and Penrose 1996). In fact, this is why recent approaches to quantum gravity try to explicitly reconcile energy and information. This is particularly apparent when dealing with black holes. But there is still another point to this, constituting our next conclusive remark.

Second, as is obvious from the underlying intention of unified theories, they also refer to a kind of secularized grounding problem which in metaphysical philosophy is traditionally dealt with when talking about the concept of substance and its attributes (Zimmermann 1991; 1998a; 2005a; 2010; 2011). From time to time this perspective is mentioned more or less at random, but altogether, the philosophical perspectives taken by physicists are very often far from being relevant and precise. This is mainly due to the terminology utilized according to somewhat arbitrary criteria and to the mixing up of ontological and epistemological problems. First of all, there are serious attempts to conceptualize the underlying physics with a view to basic principles which give a kind of philosophical grounding to physics normally absent when discussing physical details. Roger Penrose, for example, in his 1995 Tanner Lectures, is comparatively prudent in his formulations when stating that "[w]hat we need is a criterion to enable us to estimate when two space-times differ significantly and this will lead to a time-scale for Nature's choice between them. Thus, the viewpoint is that Nature chooses one or the other according to some rule we do not understand yet" (Penrose 1997, 86). In that case he points to the theory of consciousness which he has developed himself together with Stuart Hameroff. Therefore, for him the solution must be somewhere in the quantum domain: "It seems to me that consciousness is something global. Therefore, any physical process responsible for consciousness would have to be something with an essentially global character. Quantum coherence certainly fits the bill in this respect." (Ibid., 133) He thus concludes that "[m]entality ... [is] ontologically fundamental in the Universe" and mentions some kind of "proto-mentality" (Ibid., 176). This is something we can subscribe to: If there is a choice for Nature, then Nature is acting in a sense, it is subjective rather than objective. This is indeed an idea that is present in philosophical theories from Schelling to Bloch. And in particular it is the idea of characteristic time-scales that fits nicely to Schelling's worldly epochs (Zimmermann 1998a; 2004b; 2010; 2011). Pitifully other physicists do not share Penrose's modesty; for instance, Lee Smolin claims that "[p]hysics should be more than a set of formulae that predict what we will observe in an experiment; it should give a picture of what reality is .... It cannot be that reality depends on our experience" (Smolin 2006, 7). As to the first statement we notice that the vagueness in formulation actually destroys the strong argument provided by Penrose, because automatically, we envision a world which is some sort of living creature and loose the aspect of "proto-entities"

On the other hand, the vagueness in Smolin's statement renders the whole approach to end up with a false idea. This is mainly so because it is not quite the task of physics to say what there actually is. And it is a mere claim that reality cannot depend on our existence (because it is this very reality that produced us in the first place). But the main point is here that the concept of reality is far from clear: because traditionally, reality refers to what the world is like in absolute truth but that we cannot actually perceive at all, because the cognitive capacity of human beings is limited. Hence, the world as we see it is its modality, the world as it really is we call reality. Obviously, the former can only be an approximation to the latter. And this is what in the physics of quantum gravity we would also like to call approximation or emergence (Ashtekar 1998, 181; Johnson 2002; Davies 2004, 10; Deutsch 2004b, 93, 100; see also: Deutsch 2004a; Penrose 2005, 603). Hence, in the 
strict sense of the concept, Smolin would be right (but then, physics could not help). Or, if he has mistaken the concept and refers to modality instead, he is simply wrong, because the latter does indeed depend on our existence. A similar critique is adequate when referring to the recent book of Vedral's (2010) when the author struggles with the concepts of nothingness and creatio ex nihilo (Ibid., 2, 5). In fact, he does not actually answer the questions he starts with ("... why is there a reality at all and where does it come from?"), because on the one hand, he shares with Smolin the same difficulty referring to the correct meaning of "reality", and because on the other hand, he forgets that information (as well as energy) is a worldly concept which is utilized for human modelling, but not part of reality proper.

Now, in order to summarize, we can say the following. Similar to the concept of energy, information is already always present in fundamental physics. Both energy (and the matter which it is manifesting) and information are two different aspects of the same underlying primordial structure of the world we will know not before there is a consistent TOE. Within this theory, both concepts have to be unified, and by doing so, there will also enter the aspect of some cognitive meta-theory which tells us how human modelling is coming about as part of a process actually performed by nature. Such an approach will also establish an innovative relationship between philosophy and the sciences, because epistemologically, all of them have to rely on each other. Hence, the appropriate TOE cannot be found, if a philosophical framework for the grounding of the world is not also developed, which in turn is only possible if philosophical research gets interdisciplinarily entangled with the other fields of the sciences. What this attitude is actually up to shall be discussed in this present paper.

\subsection{The General Definition of Information Revisited}

Much of what we have said so far is the foundation for the results announced earlier, which generally point to the role already attributed to the concept of information when applied within the framework of fundamental physics. So, Floridi is certainly right when mentioning that "information can be said in many ways just as being can" and that "th[is] correlation is probably not accidental" (Floridi 2004, 40). But it is not clear why a UTI project should be necessarily reductionistic, because - different from the unified projects in physics - it deals with a conceptual rather than physical unification; primarily it aims at a conceptualization which is for information what it was before for energy and mass. In other words: Unification means here unifying energy and information rather than unifying different types of information. Hence, it is also a project of unifying a catalogue of terminologies, but at the same time one of unifying two irreducible phenomena. Similar to quantum physics where the difficulty is to distinguish between what is axiomatic and what is empirical, modern information theories have to distinguish between what is substantial and what is accidental. (This is summarized somewhat in Capurro's Trilemma, §1). In the case of quantum physics, the result is a bundle of interpretations, and it is hoped that eventually it will be possible to settle on a master interpretation. In the case of information, the task is practically the same. The crucial difference may be the fact that certainly, an adequate UTI will not be grounded on the mathematical theory of communication in the sense of Shannon, but will instead turn out to be part of a physical TOE. It is, however, all the more necessary to determine what meaning is all about, a notion which according to common terminology surpasses the concept of mere information, which is linked to Floridi's remark on the advent of hermeneutic theories (Floridi 2004, 41). Nevertheless, it is insufficient to let things be as they are without going into further detail as to a possible definition of the underlying basis of meaning. (See also Floridi 2010; 2010b; 2011a-b).

This is mainly so because the concept of meaning enters the discussion very early. Starting with a proper definition, it is immediately part of what Floridi calls the General Definition of Information (GDI). This definition states that an instance of information visualized as objective semantic content is given, if and only if (iff) it consists of $n$ data $(n \geq 1)$ which are well-formed and meaningful. (Floridi 2004, 42 - our emphasis) There we are: from the beginning on we have to deal with meaning. And having a look at the list of possible data within the definition's range (lbid., 42 sq.), we notice that primary data, metadata, as well as operational data can be found throughout nature: they are not restricted to social systems. Only derivative data extracted from the first three types are possibly reserved for social systems. The question is whether this is also true for meaning. If there is no information without data representation, and if a datum is a relational entity (Ibid., 43), then obviously, throughout nature, there is information which by its very relational quality always entails meaning in the first place. This does, however, imply a significant difference between what Floridi means by meaningful data and what we mean by stating the intrinsic meaningful value of data itself, as we have argued in detail elsewhere (Zimmermann \& Díaz Nafría 2012). 
If Bateson is right, and information is the difference which makes a difference (Floridi 2004, 44), then it is quite straightforward to notice that the existence of a difference immediately implies the means of recognizing a difference as difference, in other words of interpreting differences (Zimmermann 2007a; Zimmermann \& Soci 2004). This is so because the mere existence of such relational difference in reality implies that the informee - as a part of reality too - can likewise reflect a similar or corresponding relational difference so as to detect it whenever it gets in touch with the former difference. We can also say, there is a minimal nucleus of proto-type forms of cognition and communication essentially comprised of a detection device which is able to distinguish between what a signal actually shows and what this actually means (Díaz Nafría 2011) - independent of whether the physical structure (be it a living structure or not) is able also to reflect about the fact that presently, it possesses knowledge of this process.

As to the concept of meaning we find this line of argument confirmed from time to time, if often only as a side-remark. Seth Lloyd, for example, is quite clear about meaning: "If you adopt Wittgenstein's perspective that the meaning of a piece of information is to be found in the action this information provokes, the meaning of a computer program written in a particular computer language is to be found in the actions the computer performs as it interprets that program" (Lloyd 2006, 26). And we remember that action - according to quantum gravity theory - is already there from the very beginning. A spin network, the fundamental fabric of space, processes the information which is produced by means of the organizing action of the loops co-operating in order to constitute the network in the first place. This has an interesting consequence: a loop in the above sense fulfils what Stuart Kauffman calls the criteria for autonomous agents, namely, the ability to perform full thermodynamic work cycles for the provision of its own needs and the participation in natural games according to the constraints of its environment (Kauffman 2000-2006). This aspect has already been mentioned in the recently emerged field of biosemiotics (Taborski 1999; Zimmermann 2007a; Hoffmeyer 2010, 192).

Hence, although we can live with Floridi's formulations of Ontological Neutrality: ON 1 through 4 with respect to adequate data representations (Floridi 2004, 44 sq.), we dispute formulation GeN Genetic Neutrality, i.e. data can have a semantics independently of any informee - and also the viewpoint that false information is no information. In other words: we would like to stay with the GDI, but would prefer to choose another interpretation of some of its consequences.

In fact, what we would aim for can be illustrated in more detail when looking at the catalogue of main concepts assembled in the handbook edited by Floridi: beside information, there is computation, complexity, and system. From elaborating on the first (Copeland 2004) we obtain the importance of Goedel's theorem which restricts the power of computability from the beginning on. Complexity however, is visualized exclusively as computational complexity which is probably a little too narrow (Urquhart 2004). Finally, Mainzer (2004) is quite correct in stressing the origin of systems which is in dynamical systems (in the mathematical sense). The importance of this insight lies in the idea that one cannot describe any dynamical system without describing its state space at the same time. And as we know from more recent developments, the KAM (Kolmogorov-Arnold-Moser) theorem points to the ubiquity of mixed systems such that dynamical forms of deterministic chaos dominate the processes throughout nature (cf. ibid., 31). Now, if structures in nature and society can be explained by the dynamics of complex systems and their attractors (Ibid., 33), then indeed, the existence of observable structures is essentially grounded in their underlying information: Hence, "[a] dynamical system can be considered as an information processing machine, computing a present state as output from an initial state of input. Thus, the computational efforts to determine the states of a system characterize the complexity of a dynamical system." (Ibid., 36) The point is here that as far as computation goes, this formulation is certainly correct. But in view of the Goedel theorem, computational complexity is not quite satisfactory after all. The solution may be found in what Mainzer calls "computational ecologies": possibly, it is self-organizing agents as they are already available in computer networks which open a new perspective here. (See also what we said above on S. Kauffman's autonomous agents.) But then, game theory becomes relevant again (Jantsch 1982; Zimmermann 2004a; 2005; 2006).

Hence, we can state that the GDI is confirmed with respect to the emergence of meaning which is visualized as a concept to be handled parallel when dealing with information: Information is always meaningful, and it is the emergence of an autonomous agency within a particular context that comprises at the same time: meanings (as the courses of efficient and functional actions with respect to eventual interactions within its context, embodied in constraints that enable the driving of work) and information (as what enables the selection of courses of action for both the fulfillment of agent's needs and the participation in natural games within its context). On the other hand, the GDI has to be modified with respect to false information and meaning, as we have discussed in detail 
elsewhere (Zimmermann and Díaz Nafría 2012). The idea is that also false information can be utilized for a productive purpose, not only in the case of biological systems (when a copying error of the DNA produces a mutation which may be able to survive and even grow), but also for the case of fundamental physical systems (the difference being that copying errors in spin foams have not yet been studied sufficiently so as to determine what a surviving mutation would be in practice).

\subsection{Emergence of Meaning}

Hence, the Universe is meaningful from the beginning. Meaning emerges alongside with information, together with energy, at the Big Bang. Subsequently, the evolution of meaning is characterized then by emergent steps in the development of complexity. The preliminarily crucial step is being initiated by the emergence of reflexive (or: self-reflexive) meaning as exhibited by human beings (cf. Crutchfield 1994). But, how does emergence actually work? Emergence can be best visualized as emergence of averages, very much in the classical, statistical sense. For a given system, macroscopic phenomena are then nothing but approximations of processes taking place on the microlevel of state space. The former are essentially observable, the latter are essentially non-observable. Hence, in contrast to Floridi's observables, we consider these not as pure epistemological category, rather as something ontological in the first place, namely an ontological emergence determined by the interactions at its lower level of complexity; thus, in clear opposition to Floridi's explicit rejection of ontological levelism (Floridi 2011b, 47). However since observation happens at a given level of interaction, observables are also epistemic. Therefore to this respect, our stance is onto-epistemic, as stated above.

And why do we think to conceptually solve Capurro's trilemma $(\S 1)$ then? Because it is the evolution of complexity (as related to Stuart Kauffman's $4^{\text {th }}$ law of thermodynamics) that demonstrates that the multiperspectivity of univocity, analogy, and equivocity, respectively, does not actually present a trilemma. Instead, it unfolds the local perspective of conceptualization with respect to that level of complexity which is topical for a given discussion. E.g., if asking what the difference of selforganized non-living and living matter can mean (as Wolfgang Hofkirchner asks in Ibid., 24), the answer is simply that it is the level of complexity which gives a ranking to structures (indicating a lower or higher rank in the state of organization, in fact). Hence, as we deal in physics with one definition of energy plus a conservation theorem (overall balance), but with various forms of energy which are permanently transformed into each other (defining various balance equations such as that which describe the fine structure of the mentioned conservation theorem), we equally deal in the theory of information with one definition of information plus a set of evolution theorems (the four laws of thermodynamics), but with various forms of information which are also permanently transformed into each other: The essential idea (capable of achieving a broad consensus) is that in physics, energy is in some sense the prime expression for the potentiality of a system. As McMullin says: "It almost seems that it is to the potential, rather than to the actual, that reality should be attributed at the most fundamental level" (McMullin 2010,33). This is in fact compatible with quantum theory. As Jeffrey Bub (1997) has shown, the Schroedinger time-dependent equation characterizes the temporal evolution of what is possible, not what is actual at time t:

"II]n a classical world, change is described by the equation over time of what is actual, where what is actually the case ... is selected by ... the classical state - as a temporally evolving substructure against the background of a fixed Boolean lattice of possibilities. In a quantum world, what is actually the case at time $t$ is selected ... on a changing background of possibilities. So in a quantum world there is a dual dynamics: the Schroedinger dynamics for the evoIution of possibility, and a dynamics for how what is actually the case changes with time ... From this perspective, we can understand the phenomena of interference and entanglement ... as arising from the way in which what is actually the case at $t$ changes from $t$ to $t$ ' in such a way as to mesh with the change in possibility structure from $t$ to $t^{\prime}$. ... I still think the essential difference between classical and quantum mechanics is captured by the insight that going from classical to quantum mechanics involves the transition from a Boolean to a non-Boolean possibility structure for the properties of a physical system." (Bub 1997, xii, xv; cf. Magnon 1997).

We would like to claim a similar differentiation for the concept of information because it is wellknown that there is a generic difference between information about what is actually the case, and information about the possibility for something to become the case eventually. In fact, comparatively early, von Weizsaecker (1971) has mentioned a similar aspect when defining energy as the potential to move matter and differentiating information from both matter and energy (ibid., 344 sqq.). For him, information shows up as a measure for the quantity of form (complexity?) and can be de- 
termined by the number of single alternatives which have to be decided in order to describe the form (Ibid., 347). This opens interesting points for discussion, although we would not really ascribe to his equations matter, motion $=$ form, mass $=$ information = energy, in the end (Ibid., 361). But we can clearly recognize that the relevant discussion has been begun much earlier than usually noticed. Jantsch refers to von Weizsaecker in his work from 1982 (Jantsch 1982, 88 sqq., also 202). In Jantsch, contrary to von Weizsaecker, information is made somewhat more precise when discussing the co-evolution of macro- and microlevels as origin of complexity. Information then (information) refers to a special dynamical regime of a self-organizing structure. (Ibid., 300) Furthermore, by using the aforementioned Weizsäcker's distinction between potentiality and actuality of information (Weizsaecker 1971, 28), we would like to generalize this distinction, ascribing, on the one hand, potentiality to energy and information, with respect to the realization of changes or the selection of changes respectively; on the other hand, actuality to matter and structure with respect to the actualized changes and the selected changes respectively.

\section{The Regressive Perspective: Bottom-Up}

\subsection{Manifestation of Reality as Emergence}

As we have seen in previous sections, at each level of the hierarchy of complexity the cooperating parties produce an action whose course constitutes the meaning of the corresponding agency. This meaning produces in nature new regularities, new classicities in the upper levels, which are emergent in both ontological and epistemological senses; ontologically emergent, because they represent properties which are not reducible to the mere superposition of the properties of the involved parties, but essentially dependent on the rules of interaction; epistemologically, because these regularities constitute the environmental uniformities that agents - at the macrolevel - can sense.

Although we might consider different kinds of sensing, vision constitutes a paradigmatic and highly developed way of sensing the environment, quite widespread throughout animal species. It entails the reception of the electromagnetic radiation coming from objects which generally scatters an illuminating homogeneous radiation (at least homogeneous in comparison to the heterogeneity of the scattered radiation). Abstracting the means of sensing this radiation, we can regard this scattered field surrounding the object as the manifestation of the object itself or as potential observation, which is indeed emergent to the underlying reality - as we will see by analyzing the nature of such radiation. This emergence, in which the reality causing the actual manifestation is contingent to the manifestation itself (i.e., it can be produced by an open set of equivalent objects) imposes on the subject of observation an ontological boundary with obvious epistemological consequences. Further epistemological boundaries are given by limitations of the electromagnetic sensing apparatus of animal vision.

\subsubsection{Physical Limitations of the Manifestation of Reality}

Although the normal case of observation is constituted by the scattering of an illuminating radiation, the problem of perception is actually related to the attention on the heterogeneities due to the scattering field, therefore it is reasonable to focus on the equivalent problem of observing a set of electromagnetic radiating sources - avoiding the need to consider illumination. If we hypothetically knew the set of radiating sources, the question of how they manifest over a domain of potential observation $\mathcal{D}$, as illustrated in figure $1 \mathrm{a}$, can be directly handled by using the Maxwell equations. The linearity of these equations straightforwardly allows us to apply superposition in order to find out the field distribution over the domain of interest. This problem is commonly called the "forward problem". However, the problem of perception is opposite: the field distribution over an observation domain - the retina - is to some extent given, while the related source distribution is intended. This is usually referred to as the "inverse problem". According to the electromagnetic uniqueness theorem, there is a unique solution for the field distribution surrounding the sources whenever either the electric or the magnetic field is given at any surface enclosing the sources, for instance at surface $S$ in figure 1 (cf. Balanis 1989). Hence, there is a degree of freedom corresponding to which surface is selected; in other words - as could also be argued using Schelkunoff's equivalent theorem (1936) - a volumetric distribution is undetermined by a surface distribution. The contingency of the actual source distribution with respect to its manifestation constitutes a basis for speaking of manifestation as emergence: it is the co-operation of the parts related to the organization of the field produced by each part which manifests as a whole. This represents an ontological limit directly related to the epistemological boundary of delving into the object enclosed by $S$. 


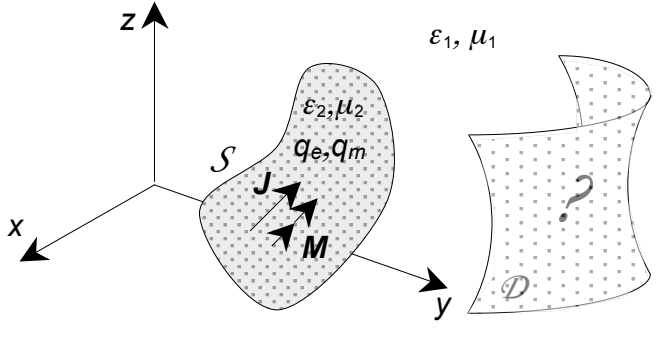

a) Forward Problem

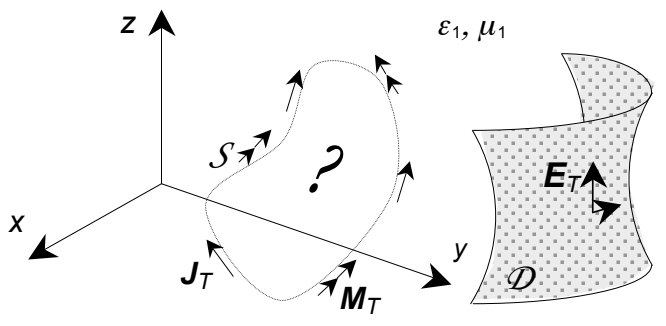

b) Inverse Problem

Figure 1: a) In the forward problem, the linearity of the Maxwell equations provides a straightforward solution; b) In the inverse problem, the uniqueness and equivalent theorems limits the problem to the determination of a superficial distribution.

The feasibility of solving the inverse problem can be handled in terms of the complexity of the information provided by the electromagnetic fields generated by the object. In fact, the complexity of an electromagnetic field of wavelength $\lambda$ is strictly limited - as one of us has proven elsewhere (Díaz Nafría and Pérez-Montoro 2011b; Díaz Nafría 2003, 2011):

(i) There is a minimum distance between independent intensity values, which is $\lambda / 2$ for an arbitrary observation (sampling theorem for arbitrary observation) and $\lambda d / 2 a$ for observation at a distance $d$ from an object within a ball of radius a (sampling theorem for distant observation). This minimum distance can also be regarded as the size of the smallest perceivable details (or heterogeneities).

(ii) The maximum Kolmogorov complexity of the field produced by a source within a ball or radius a is limited to $N=32 \pi(\tau a / \lambda)^{2}$ (complexity theorem for radiation field), where $\tau \geq 1$ stands for an excess of the maximum spatial frequencies with respect to $2 \pi / \lambda$ at $S$, related to the relative presence of evanescent modes at the vicinity of the object.

Concerning this maximum complexity, it is interesting to point out, on the one hand, that no matter how big the complexity of the object is, the complexity of the field distribution surrounding the object cannot be bigger than $N$; on the other hand, that such complexity depends on $a^{2}$, thus on the area of the surrounding boundary, not on its volume. Consequently, the ambiguity provided by the radiation of the object with respect to its volumetric structure corresponds to one dimension. ${ }^{1} \mathrm{Nev}$ ertheless, though $N$ constitutes a natural limit to what can be given by the field, generated by an observed object, this is just a maximum rarely reached by such field, and - what is more important to the problem of figuring the object from its manifestation - by the complexity of the object itself, say, $N_{O}$. It is clear that if $N_{O}>N$, finding out the structure of the object from its field is out of reach, the question is then whether the observation is enough for finding out the structure of the object in case of $N_{O}<N$.

This consideration of the manifestation of an object in isolation, independent of the observer, should not be interpreted as a pure realist or objectivist stance. It is indeed the interaction with the environment that is here considered since the space where the electromagnetic field takes place is much more than nothing (in the sense of ontological emptiness), it has a structure which can be expressed in terms of electric permittivity and magnetic permeability. With respect to the observer, the validity of our classical electromagnetic analysis requires that the observer has little effect on the whole field distribution. Therefore, the field distribution around an isolated object can be regarded - under this assumption - as potential observation.

\subsubsection{Limitations of the Sensing Apparatus}

Whilst the aforementioned limitations are independent of any sensing ability, it is also worth considering how the sensing structure of animal vision is adapted: on one side, to the physical limitations of the electromagnetic radiation; on the other, to the leeway and constraints offered by the evolutionary path, as can be - for instance - observed through comparison between vertebrate and cephalopod vision. This viewpoint represents a significant difference to Floridi's account of data and the Levels of Abstraction (which are in turn constituted by observables): whereas in Floridi

\footnotetext{
${ }^{1}$ Though for the scattering problem - i.e. the usual case of observation -, this might be considered trivial, the problem, as stated, comprises a volumetric distribution of electromagnetic currents.
} 
these are given, we try to explore what can be regarded as its emergence - as we have discussed in detail in (Zimmermann \& Díaz Nafría 2012).

Comparing the physical limits of the electromagnetic fields stated above with the structure of the retina, we observe that the distances between photoreceptors are within the boundaries stated by the aforementioned limit (i): whereas the minimum expected distance between independent values of the electromagnetic field is $0.2-0.4 \mu \mathrm{m}$ for visible spectra $(400-800 \mathrm{~nm})$, the minimum distance between photoreceptors (corresponding to its maximal density at the fovea of human retina) is $2.2 \mu \mathrm{m}$ on average, and $1 \mu \mathrm{m}$ for animals with maximal visual acuity (some birds), which clearly does not surpass the physical limits (Curcio et al. 1987). Moreover we might ask why the vision apparatus of some species does not appear to have evolved to reach the physical limit - particularly since it could provide an adaptation advantage. To find an answer to this reasonable question, we should consider at least two important constraints of vertebrate vision:

(1) Dispersion at the photoreceptors due to the nervous network located over the photoreceptors layer as shown in figure 2.a (which is the most common case for camera-type eyes, though not for cephalopods for instance, which vision - figure $2 . b$-, though functionally similar, followed a different evolution path with respect to vertebrates since about 600 million years (Lamb 2011; Ogura 2004));

(2) Spherical aberration, due to the roundness of the eyeball structures, which therefore decreases if the eye size increases.

Besides fine disturbances due to the former, its weight clearly increases if the eye size also increases (since dispersion happens through a longer distance); therefore both constraints impose an antagonist pair which distances vision acuity from the possibility of perceiving the heterogeneities actually present in the electromagnetic field. As argued in (Díaz Nafría 2008), the peculiarities of bird vision probably allows a best compromise in which the minimum distance between independent values of the field at the photoreceptors layer is about $1 \mu \mathrm{m}$. But, beyond this relative optimal, the question is why vertebrate vision did not evolve as in the cephalopods, locating the photoreceptors above the neuronal network. To this respect Lamb's hypothesis (2011) offers a suggestive explanation:

Animal photoreceptors are either of rhabdomeric- or ciliary-type. The former are common in invertebrate, the latter in vertebrate vision. However, ciliary-type photoreceptors are also present in most organisms for non-visual purposes (sensing light for regulating circadian and seasonal rhythms), whilst rhabdomeric cells subsist in vertebrates, though transformed into projection neurons. By means of primitive evolution of vertebrates, in abyssal dark environments, the photosensitive rhodopsin of ciliary photoreceptors underwent a change conferring to these photoreceptors higher sensitivity than is achieved by rhabdomeric ones. This advantage allowed the colonization of dark ecological niches (probably just for circadian and seasonal regulation at the outset). In this context rhabdomeric photoreceptors adopted a new role: transmitting and processing signals to the brain. Since - in the former topology - these photoreceptors were directly located where the light comes from, this topology imposed a constraint that could not be reverted causing that the neuronal network was developed above the photoreceptors and therefore producing the aforementioned drawback (1). Nevertheless, the advantage provided by the evolution in sensitivity is clearly expressed by the fact that vertebrate rods can detect single photons, therefore reaching the strict physical limit to this respect. 
a) Retina structure of vertebrates

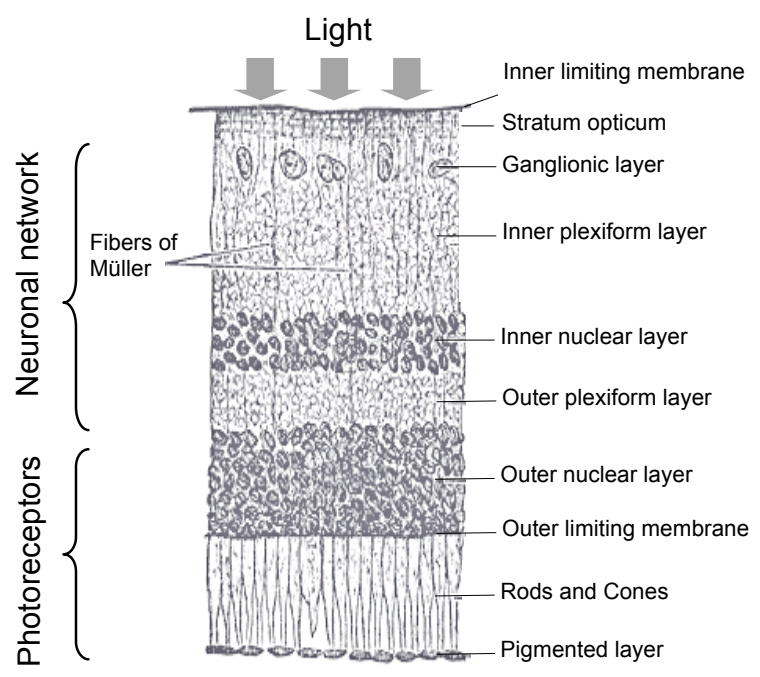

c) Retina structure of octopus

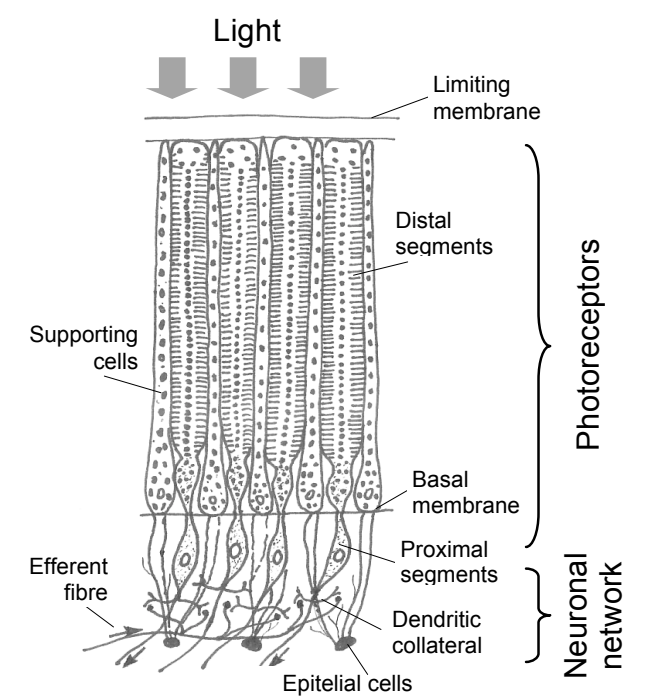

Figure 2: In vertebrates, the light must pass through the transparent neuronal network causing dispersion before it reaches the photoreceptors (of ciliary-type), whilst in the retina of the cephalopods the photoreceptors (of rhabdomeric-type) are immediately under the membrane limiting with the vitreous body. [The illustration has been elaborated using: a) fig. 881 of Gray's Anatomy (1918); and b) own artwork based upon description of cephalopod's retina from Young (1962)].

But coming back to the fact that visual acuity - even in birds - is somehow distant from the potential manifestation of reality, analyzed in the previous section, we can state that given the constraints of the evolving structure, the sensing apparatus agrees with the maximal heterogeneity that such type of structure can get to detect.

Another major limitation of animal vision concerns:

(3) The lack of sensitivity to phase variations of the received field, which implies - as one of the authors has shown (Díaz Nafría 2003) - the impossibility of determining a unique distribution over a surface bounding the object from the observation at just one surface (e.g. the retina of one eye).

To this respect, it is interesting to notice that natural evolution has solved this constraint through combination of two eyes, even though camera-type eyes probably evolved from the pineal gland, thus without bilateral symmetry at the outset. (This point has been discussed in more detail in Díaz Nafría \& Zimmermann 2012)

\subsection{Emergence of Intention: Closing the Hermeneutical Cycle}

Turning back to the analysis of the physical problem as stated in $\S 3.1 .1$ : in the case of $N_{O}<N$, the observation of the object could be enough - from the viewpoint of the amount of information needed - for determining a proper idea of its volumetric distribution. However, since there is in principle an unlimited number of inner structures whose projections over a bounding surface are equivalent, as well as an undetermined number of projection surfaces, such an 'idea' (or model of the observed reality) should be achieved based upon some guesses, assumptions or a priori knowledge of the inner structure. These can be interpreted as the semantic or algorithmic ground for reconstructing the object, in the sense of the algorithmic information theory (Burgin and Díaz Nafría 2011), but considering the evolution of these semantic grounds, it can also be visualized in terms of Thom's logoi dynamics, as the authors have argued elsewhere (cf. Zimmermann and Díaz Nafría 2012, §5.2; cf. also Zimmermann 2001).

However, disregarding this evolutionary perspective of interpretation, to which we will come back later, the limits of interpretation can be better analysed by properly posing the problem of observing an object within a bounded region, and assuming that the interaction level in which ob- 
servation takes place can be well described by Maxwell's relations, to which also the previous physical analysis (§3.1.1) refers. This corresponds to an idealised situation, but, on the one hand, natural observation tends to it (as we proved above); on the other, it serves to evaluate the limits of what natural observation can achieve.

\subsubsection{Physical Limits of the Meaning-Offer}

According to theorems (i) and (ii) together with the aforementioned equivalent theorem (Schelkunoff 1936), it can be shown that a useful way to make the inverse problem well-posed is by locating $N$ equivalent tangent point sources over $S$ regularly spaced at a distance $\lambda / 2 \tau$ :

$$
\hat{\mathbf{s}}(\mathbf{r})=\sum_{i=1}^{N} \hat{\mathbf{s}}_{i} \delta\left(\mathbf{r}-\hat{\mathbf{r}}_{i}{ }^{\prime}\right)
$$

where is here used to indicate estimates for the equivalent variables corresponding to the model of the object: $\left\{\hat{\mathbf{r}}_{i}{ }^{\prime}\right\}$ is the set of locations of the equivalent point sources, and $\hat{\mathbf{s}}_{i}$ represents the intensity of an equivalent point source situated at $\hat{\mathbf{r}}_{i}{ }^{\prime}$. The space of equivalent manifestations,

$$
\hat{\mathbf{\Psi}}(\mathbf{r})=\mathbf{G}(\mathbf{r}) * \hat{\mathbf{s}}(\mathbf{r})=\sum_{i=1}^{N} \hat{\mathbf{s}}_{i} \mathbf{G}\left(\mathbf{r}-\hat{\mathbf{r}}_{i}{ }^{\prime}\right)
$$

generated by the space of equivalent source distributions $\{\hat{\mathbf{s}}(\mathbf{r})\}$, is equivalent to the set of eventual manifestations of any arbitrary inner (discrete or continuous) volumetric distribution. (In the appendix, some details are provided about how to interpret these mathematical entities physically, as well as how to derive them from the Maxwell relations).

For the sake of simplicity we may suppose that the real source (i.e. the observed reality) is described by a set of $N_{O}$ Dirac delta distributions of different amplitude and position within the volume enclosed by $S$ :

$$
\mathbf{s}(\mathbf{r})=\sum_{i=1}^{N_{O}} \mathbf{s}_{i} \delta\left(\mathbf{r}-\mathbf{r}_{i}{ }^{\prime}\right),
$$

whose corresponding manifestation is given by:

$$
\boldsymbol{\Psi}(\mathbf{r})=\mathbf{G}(\mathbf{r}) * \mathbf{s}(\mathbf{r})=\sum_{i=1}^{N_{O}} \mathbf{s}_{i} \mathbf{G}\left(\mathbf{r}-\mathbf{r}_{i}{ }^{\prime}\right)
$$

Despite the formal similarity of (2) and (4), it is worth emphasizing the relevance of the differences $N$ vs. $N_{O}$, and $\hat{\mathbf{r}}_{i}{ }^{\prime}$ vs. $\mathbf{r}_{i}{ }^{\prime}$. Whereas the former makes (2) directly related to the maximal complexity of the field distribution $N$; the proper selection of $\left\{\hat{r}_{i}{ }^{\prime}\right\}$ (regarding regular distancing and coverage of $S$ ) warranties the independency of the fields generated by the equivalent sources. Thus, (2) is invertible, but there is no guarantee about the invertibility of (4). Furthermore, since $\boldsymbol{\Psi} \in\{\hat{\boldsymbol{\Psi}}\}$, it is possible to determine a unique equivalent distribution belonging to $\{\hat{\mathbf{s}}\}$ and compatible with $\boldsymbol{\Psi}$ :

$$
\hat{\mathbf{s}}\left(\hat{\mathbf{r}}^{\prime}\right)=\Psi(\mathbf{r}) * \mathbf{G}^{-1}(\mathbf{r})
$$

\begin{tabular}{|c|c|c|}
\hline Reality & $\mathbf{s}(\mathbf{r})=\sum_{i=1}^{N_{O}} \mathbf{s}_{i} \delta\left(\mathbf{r}-\mathbf{r}^{\prime}\right)$ & \\
\hline $\begin{array}{l}\text { Information } \\
\text { (manifestation) }\end{array}$ & $\boldsymbol{\Psi}(\mathbf{r})=\mathbf{S} * \mathbf{G}(r)=\sum_{i=1}^{N_{O}} \mathbf{s}_{i} \mathbf{G}\left(\mathbf{r}-\mathbf{r}_{i}^{\prime}\right)$ & $\hat{\boldsymbol{\Psi}}(\mathbf{r})=\hat{\mathbf{s}} * \mathbf{G}(r)=\sum_{i=1}^{N} \hat{\mathbf{s}}_{i} \mathbf{G}\left(\mathbf{r}-\hat{\mathbf{r}}_{i}^{\prime}\right)$ \\
\hline \multirow[t]{2}{*}{$\begin{array}{l}\text { Meaning- } \\
\text { offer }\end{array}$} & & $\hat{\mathbf{S}}\left(\left\{\hat{\mathbf{r}}^{\prime}\right\}\right)=\sum_{i=1}^{N} \hat{\mathbf{S}}_{i} \delta\left(\mathbf{r}-\hat{\mathbf{r}}^{\prime}\right)=\boldsymbol{\Psi}(\mathbf{r}) * \mathbf{G}^{-1}(\mathbf{r})$ \\
\hline & $\begin{array}{l}\text { Reality and Manifestation } \\
\text { of the object }\end{array}$ & $\begin{array}{l}\text { What is unambiguously offered to } \\
\text { the subject concerning the object }\end{array}$ \\
\hline
\end{tabular}

which - as illustrated in Figure 3 - can be conceived as the meaning-offer of $\boldsymbol{\Psi}$ upon the semantics described by (1) and (2). 
Figure 3: On the left: spaces of (supposed) reality and manifestation of the object, when the complexity is constrained to $N_{O}$ punctual heterogeneities; on the right: spaces of manifestation (or information) and meaning-offer on the observer (subject) side, whose complexity is constrained to $N$ punctual heterogeneities. The real structure of the object (here determined by $N_{O}$ values and positions) remains veiled to the subject, whereas a projection in the space of $N$ punctual heterogeneities can be achieved.

\subsubsection{Unveiling Reality: Hermeneutical Agency}

But returning to the case in which $N \gg N_{O}$ - which is a rather typical case if we disregard small scale heterogeneities, and consider the low entropic objects we usually deal with - the real complexity of both the object and its manifestations is much smaller than the complexity corresponding to equation (5), then some representation could be found in which the description becomes shorter. The simpler the description, the better it can be extracted from noise and therefore it is received cleaner. Nevertheless, it is well known that - according to Turing's halting theorem - there is no recursive method to decide if the minimum description has been achieved. It is thus a question of proper guessing, of finding out a proper semantics which allows the interpreter to achieve a better representation compatible with the observed manifestation. This action is carried out by an hermeneutical agent who, similarly to how nature enabled the emergence of the manifestation through the co-operation of the radiating parts, closes the cycle of interpretation by creatively constructing a possible path for the emergence of the given manifestation, though in reality it always remains open by virtue of the possibility of finding an even more efficient description. Since the hermeneutical agent is itself a part of nature, by this means, nature can recognize itself.

The fact that the real object is not merely given by its manifestation, makes the task of interpreting or modelling the object transcendental. By considering the hermeneutical subject and its activity on its material flesh as well as its hermeneutical activity we are moving within the frame of transcendental materialism as thoroughly developed by one of the authors (Zimmermann 2004b).

Given our formulation of the hermeneutical agency, it is reasonable to consider that the hermeneutical task consists of reducing as much as possible the complexity of the representation, which always remains as an open task: on one hand, because one can always seek new data about the object; on the other, because there is no sure means to know that the minimum description has been achieved for the given data.

This evolution of hermeneutic agency can be nicely exemplified beyond the case of visual perception by the historical development of the astronomical system: Tycho-Brahe's model represents an important advance with respect to Ptolemaic system by extending the observation; whereas Kepler's model represents a more efficient hermeneutic agent with respect to the former by simplifying the descriptive means, as has been discussed by one of the authors (Díaz Nafría 2011). Another interesting example for the evolution of hermeneutical agency within scientific advance (also therein discussed) is clearly illustrated by the superseding of the Aristotelian type of systems of living species (as, for example, the Linnaean taxonomy) through the evolutionary type (as in Darwin's evolutionary taxonomy).

\subsection{The Levels of Interpreting Reality}

Through evolution of complexity, the sensing apparatus increases its own complexity, which in turn causes an increase in the complexity of the related responses and representation means. As we have seen in $\S 3.1 .2$, the improvement of the sensing apparatus drives the autonomous agent towards the meaning-offer of the physical manifestation of objects, which in turn implies an increase in the ambiguity concerning the relation of what is given by sensation and what can be found out therefrom.

At a lower level of complexity the sensing apparatus offers little ambiguity with respect to what is signed. In the extreme case, minimum sensing would only sign that something has changed in the environment - which by the way constitutes the primordial datum of any sensing - though without further precision. We can also speak of minimum sensing whenever what is signed is of the kind: there is light; it is daytime; it is cold; there is too much acid, etc. In an evolutionary sense the specific sensing of the agent enables an adaptive finding of a proper "objective response" that must be stored in the organic codes - in the sense of Barbieri (2003), as clarified below. In higher complexity levels, the ambiguity - provided by sensation with respect to what is signed - increases, bringing about the need of improved means of representation and memorizing in order to solve such ambiguity, which enables the emergence of reflexive response, and hermeneutical agency. 
For the sake of clarity we can speak of four differentiated levels of response with regard to the sort of sensing the manifested reality, which can be typified as: objective, cognitive, reflexive, and socio-ethical.

\subsubsection{Objective Response}

The cell provides a paradigmatic case of objective response which is also present in systems of higher complexity - as it constitutes its basis. A cell, in general, has several means of sensing the environment and adapting to those variations which are relevant to its survival. Since we have been dealing with visual sensing, it is here interesting to consider the minimum case of seeing as represented, for instance, by the unicellular organisms of the genus Euglena, illustrated in figure 4. These cells have an eyespot apparatus which filters sunlight into the photo-sensitive structures at the base of its flagellum. This eyespot enables the cell to sense the strength and direction of light, and to move accordingly towards a medium of moderate light (away from darkness and bright light). ${ }^{2}$ The ambiguity of perception is here very low: the strength of light is high or low, and it comes from this or that direction; and accordingly the accuracy in the determination of the environmental state is low.

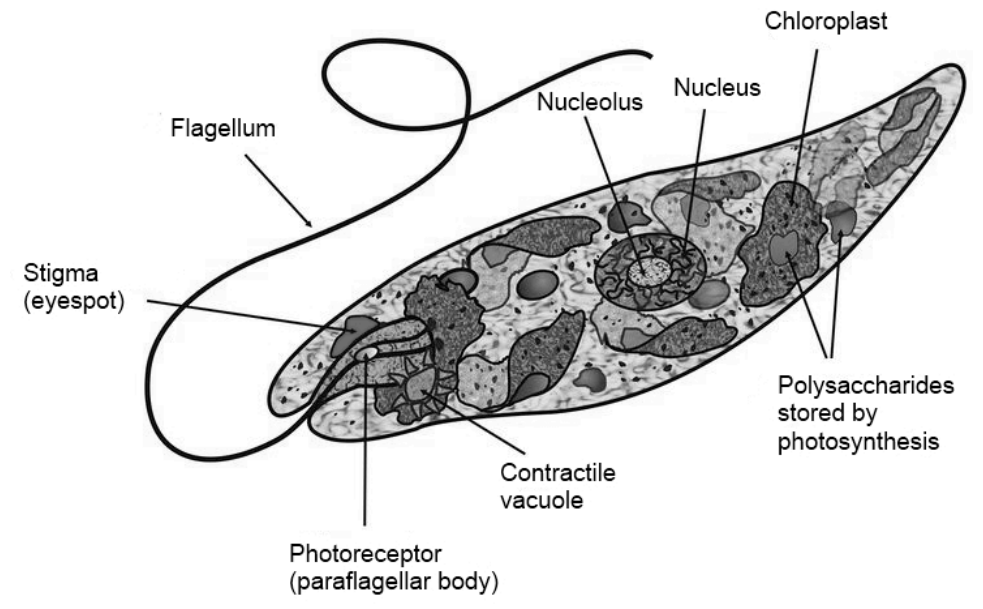

Figure 4: General anatomy of a Euglenoid cell [Illustration by C. Miklos available in Wikimedia Commons].

Generally speaking, in the objective response, the meaning is embodied in the organic structure (constituted in the Euglena by the photoreceptor, the eyespot, the flagellar swelling, the flagellum, and a contractile vacuole, linked by topologic, mechanical and chemical relations), in which a set of constraints enable an effective utilization of energy. However if - in an evolutionary sense - we observe it diachronically, these constitutive relations are established with respect to its effectiveness in offering an adaptive benefit. The dynamics of these relations (constraints for the proper driving of work) are embodied in the corresponding evolution of genetic codes - in the sense of Barbieri (2003). Genetic codes offer at the same time means of memorizing effective constraints viz. meanings - and change of these constraints for further adaptations.

From the viewpoint of our understanding of information: the light comprises in the first place besides energy - the meanings of the directivity in the driving of energy and its amount. Such meaning-offer is in itself the result of an interaction with the real space. In this respect, we can speak of first-order meaning and first-order interaction. However, this meaning-offer or first-order meanings represent a potentiality with respect to the selection of change in the cell for a proper driving of energy, which constitute second-order meanings. The action of the cell allows the actualization of its structure, which in this example implies some tropism, based upon the received information. The cell as an autonomous agent performs an effective driving of energy for the benefit of the cell in its survival. We can thus speak of proto-hermeneutics since the preliminary meaningoffer has to be actualised within the meanings of the cell, materialized in the organic structures which produce fixed actions with respect to the given interaction. Therefore, the response and its

\footnotetext{
${ }^{2}$ In the Euglena the afferent structures of the cell -sensing the environment- are directly connected to the efferent ones -the flagellum which causes the necessary movement towards a more suitable environment (PEET 2010).
} 
related meanings are objective in the sense that they comprise a fixed reaction and an objectivised mechanism of response.

\subsubsection{Cognitive Response}

In the cognitive response, the complexity grows alongside the formalisation means for the apprehension of reality, which in turn requires the ability to guess within the ambiguity given by the sensing and the manifestation of reality (as analysed in §2.1). Comparing the sensing of the cell, mentioned above, with the animal vision: both the ambiguity and the information about the observed reality increases significantly. Grasping more data about reality - particularly if they have different modality, for instance, visual and tactile data as discussed in (Díaz Nafría and PérezMontoro 2011a) - the ambiguity, left by some partial perceptions (e.g. a visual percept), can decrease although new kinds of ambiguity may appear. Probably, the completeness of percepts, through adapting new ways of sensing for given environments and given agencies, as in the case of the two eyes vision argued in $\$ 3.1 .2$ for solving the ambiguity of phase-less light reception at the retina, is evidence of perceptual evolution.

This completion of sensing by different modalities can be represented by the algorithm depicted in figure 5 (adapted from the generalized method of successive projections developed by one of the authors (Díaz Nafría 2003) for the solution of different inverse problems). Since all percepts must be consistent with the interpretation of the object, it can be shown that - in virtue of the convexity of the linear relation $\mathbf{G}_{\mathrm{i}}$, which links between interpreted objects and what is observed as described by equation (2) - the solution asymptotically converges towards a stable solution through successive and recursive application of observation-data. A tolerance with respect to the achieved stability of the solution, represented by the parameter $\varepsilon$, constitutes a pragmatic compromise which can be easily mapped in human perception. Such tolerance represents the referred open character of perception and implies a truthfulness criterion significantly different to the one proposed by Floridi in his Correctness Theory of Truth $(2011$, ch.8).

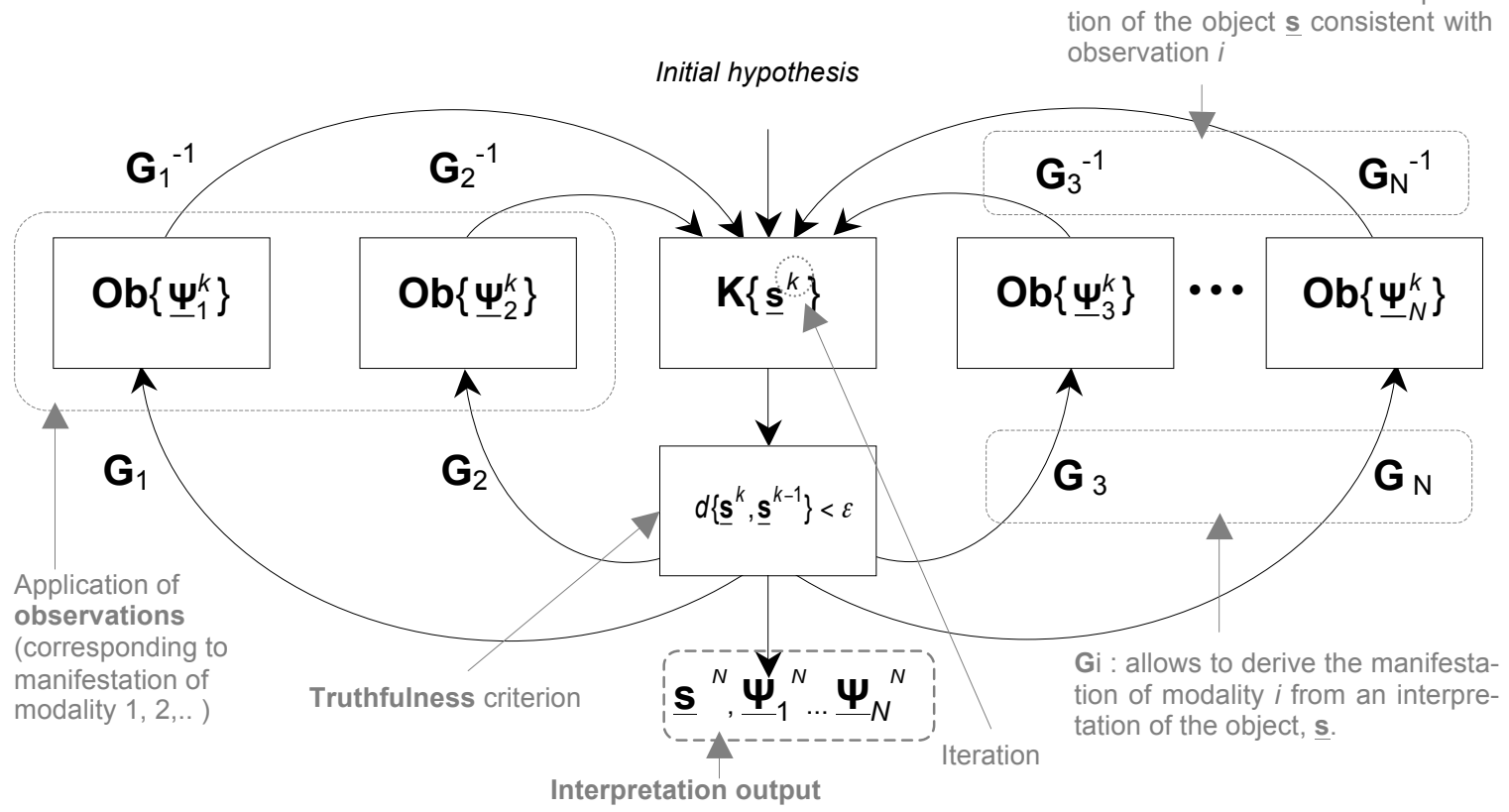

Figure 5. Algorithmic approximation to the completion of percepts by different sensing modalities (based on the method of successive projections developed for the solution of inverse problems (Díaz Nafría 2003)). Ob\{ \} represents the combination of the observation of modality $i$ with the non-observed manifestation, based on the previous interpretation of the object through $\mathbf{G}_{i}$ (which in turn links the interpretation of the object $\underline{\mathbf{s}}$ with the manifestation of modality $i$ ). Whereas $\mathbf{K}\{\}$ represents the constructed model of the object (at any iteration) through $\mathbf{G}_{i}^{-1}$ and based upon such combination of observable and nonobservable manifestations.

Unlike the linearity of relations $\mathbf{G}_{i}$ - as referred to in $\S 3.2 .1$ - in case of cognitive subjects, nonlinear relations - mediated by memory - are established between sources and phenomena, 
achieving a much faster algorithmic convergence. Furthermore, since different neuronal subsystems specialize in the response given to different sensing modes, instead of the successive application of sensing data, the cognitive response simultaneously apply different sensing modalities, which - though operative equivalent - offers an adaptive benefit regarding time-efficiency.

If we understand the algorithm here depicted as the agent activity in which actualised information (within the cognitive structure $\mathbf{K}\{\}$ ) is computed upon the information provided through observation $\mathrm{Ob}\{\}$ (including previous computations), the model offers significant alignment with the info-computationalism, as advanced by Dodig-Crnkovic (2010). However, we consider of fundamental relevance the distinction herewith established between information and energy, as well as between potential and actual information (the latter represented by structure), which in DodigCrnkovic's approach seem to be blurred.

To the issue of the actualization of the cognitive structure, at this level of complexity (i.e. higher than objective response but lower than reflexive one), neuronal-epigenesis, learning and memorizing play a significant role. Learning in the specific environments where animal life is going to be developed (often through games of immature animals) probably enables the acknowledging of relevant objects with which the animal will have to deal. By means of this acknowledgment, stored in the animal memory, the ambiguity of sensing is solved and can be directly related to a behaviour which is to a large extent determined by the genetic code (though its weight is lesser for higher animals). Therefore the apprehension of reality can be directly linked to a particular response (or, rather, to a complex set of responses), in which the efficiency of the animal agency is achieved (related to the adequate driven of energy for the animal itself). As long as the response is fixed, we cannot speak of reflexive response; to the extent that the ambiguity of the apprehension of reality is solved in the cognitive system and its related memory, we cannot speak of objective response.

\subsubsection{Reflexive Response}

In the reflexive response, since the response to the apprehension of reality is not fixed once and for all, offering through evolution a growing open character, the interpretation, though also mediated by learning (stored by memory), is left open to further revision, deepening, correction... as particularly observed in humans. It is however worth remarking that responses of objective and cognitive type - referred to above - are to a large extent present in humans. For instance, the immediate removing of the finger that is pricked by the rose spine is an example of an "objective response". But the repertoire of human responses of this kind is really extensive. Certainly, most of our somatic and visceral activity is regulated by inner and outer sensing unconsciously imposed, and frequently by means of a neuronal communication not passing through the cortex. Nevertheless, it is also a remarkable feature of our nervous system, in which evolved connexions coexist with more primitive ones, that the cortex holds the possibility of interfering with the "objective responses" though with some delay. This is because the spinal cord transmits the sense impulses simultaneously upwards and downwards (Raisbeck 1954; Sobrino and Simón 1986). We can observe this feature as a consequence of the aforementioned "leeway and constraints of the evolutionary path" $(\S 3.1 .2)$.

In any case, besides this coexistence of responses of lower complexity, it is characteristic of the reflexive response that the apprehended reality can be directly sensed as reality itself and not only as stimulation, i.e. as what produces a reaction for the adaptation to the sensed changes. Being the manifestation of reality essentially ambiguous or incomplete (for physical manifestation - as shown in $\S 3.1 .1$ - there is a degree of ambiguity corresponding to one dimension with respect to the space of representation, which can be, for instance, the four dimensional space-time) reality is sensed by the reflexive agent as fundamentally open, in two senses: (i) with respect to the object as something that has to be further fathomed, (ii) concerning its connection with the environment to which it can be bounded by different functionalities. This is particularly the case of sensing objects in cultural contexts (including its related technical means, social, as well as political and economic relations). In agreement with "perceptual functionalism" - as developed by Brunner et al. (1947) or Foerster's perceptual epistemology (Aguado 2009; Foerster 1981), we can state that perception, driven by subjective dispositions, necessities and objectives, has a sort of hypothetical character susceptible to modification, deepening and correction. But according to Gestaltpsychologie the creative abductions that are needed in the hermeneutical process, requires a structured and holistic apprehension of the interpretandum as a whole (including its connections with the environment).

The example given above (§3.2.2) of scientific discovery illustrates both the openness of hermeneutics, linked to an evolutionary arrow, and the structuration of the wholeness (in which reduction of complexity of the interpretation is a sign of effectiveness of the related agency). By this means, interpretation operates as nature: it searches for the simplest means (i.e. most effective 
with respect to used resources) for defining the agency in which the observed reality is involved. Since the course of action of the autonomous agent constitute its own meaning, when the interpretation is correct, the reflexive and the objective meanings (or their intensive and extensive aspects) agree (cf. Zimmermann and Díaz Nafría 2012, §3.2).

\subsubsection{Social and Ethical Response}

In the social response, the autonomous agent is defined by the relations established among reflexive agents, embodied roles and moral behaviour. In the emergence of social agency, there is often no reflexion on the involved benefit, though cultural semantics leave a degree of openness concerning the involved relations which enables trial and error but also free interpretation by means of which the imagination of new relations can offer shortcuts in the search of improved adapted agency. Political agency can be conceived as aligned to the latter, while many situations reported by anthropologists offer excellent examples of both. For instance, the family structure studied by Claude Levi-Strauss (1969) - particularly the Australian systems, or the interesting case of change in the ecological conditions mapped in the "Asdiwal story", reported by the same author (1967). As he analysed, a clear semantic openness was exhibited only at the time in which new relations were sought until a new ecological equilibrium was achieved. Interestingly, the semantic openness is closed through new bipolar distinctions adapted to the new relations. This points to a relevant feature of cultural symbolic universes whose meanings are adapted in normal conditions to effective agencies: offering closure - often blocking intentional agencies at the lower level - when agency is effective; exhibiting growing degrees of openness when the corresponding agency is not any more effective. Whenever the agency is stable, the meanings of the related semantic universe constitute the constraints which appropriately drive the energy to produce work in benefit of the social agency itself. Moral values constitute an important means for building constraints at this level of complexity.

Particularly in the cases in which there is an intentional search of new social agencies, it is possible to speak of self-reflexion. The subject has to interpret herself in the social wholeness. Retrospectively she can fathom her biography immersed in social, cultural and historical worlds; prospectively, she can imagine possible utopias (in the sense of Bloch) in a creative search of more appropriate agencies. This self-reflexion of the appropriate relations to drive social action constitutes the ethical reflection in itself. It aims at the objective of finding a more appropriate agency, which can be very well symbolized by the Spinozist: "The more perfection a thing possesses, the more it acts and the less it suffers, and conversely the more it acts the more perfect it is" (Spinoza 1677, V, prop.40).

\section{Conclusive Remark: Towards a Unified Perspective of Information and Meaning}

As we have tried to show there are good grounds for adopting a unified vision of these fundamental aspects of reality in coherence with our scientific knowledge. This offers - as we advanced in the introduction - a robust foothold for the development of a Unified Theory of Information (UTI) as proposed by Hofkirchner (1999; 2010). We therein stated the dependence of this success on a well-established foundation of physics, able to unify the theories of relativity and quanta. Though this has not yet being achieved, we have adopted hereby a vision which - being consistent with deep-rooted theoretical and experimental accounts - enables us to unfold an evolutionary understanding for the emergence of complexity and meaning in physical, biological, cognitive and social systems; visualising emergence in a sense that is ontological and epistemological at the same time (and can also be understood as emergence of classicities). Such insights enabled us to devise the General Definition of Information (GDI) proposed by Floridi throughout nature properly; regarding information alongside its related meaning as fundamental aspects of the structure of the world.

We estimate that the approach hereby advanced meets all the requirements of the UTI programme as enunciated by Haefner (Hofkirchner 1999, xv-sq), offering further sound foundations for its development. However, our viewpoint is not following the thought expressed by Fenzl and others that "formalism [is] of merely secondary importance" (Fenzl and Hofkirchner 1997), not because of the sheer purpose of attaining a nice formalised theory, neither for achieving quantitative scaffolds to assess how much is to gain or to lose, but because - as we have discussed through our progressive and regressive perspectives - it is the "form" (either in potentiality or actuality) that is in the core of the "new" in reality; of the emergence and dynamics of agency; of the emergence and dynamics of meaning. Therefore, formalism is of major importance whenever it aims at a proper mapping of the dynamics of form in reality, and particularly regarding qualitative features. 
With respect to the herein tackled levelism of interpretation (in which the regressive perspective has been developed), our proposal and the one developed within the frame of UTI by Fenzl, Hofkirchner and others (op. cit.; Hofkirchner 1999) are significantly aligned; but it is here worth mentioning a relevant distinction. The latter consider three fundamental levels: (i) self-restructuring, (ii) self-reproducing, (iii) self-re-creation. Whereas (i) comprises self-referential semiotics aligned to objective responses, and (iii) comprises self-anticipation semiotics aligned to socio-ethical responses; we have split (ii) - originally comprising self-representational semiotics - into cognitive and reflexive responses. This distinction is noteworthy concerning our review of GDI and the formal aspects of UTI: a) at the level of cognitive response, representation is lesser flexible, being attached to stabilized logoi - in the sense of Thom, as we have argued elsewhere (Zimmermann and Díaz Nafría 2012); b) at the level of reflexive response, the representation means are more flexible (say, $\{\mathrm{Gi}\}$ can be disputed, i.e. the relation between the alleged reality and its manifestation); thus emergence of logoi dynamics - in the sense of Thom (op. cit:; Zimmermann 2001).

But in addition to these endeavours towards a general understanding of information and meaning, there are others worth considering with a view to eventual synergies. We have previously referred to Dodig-Crnkovic's Info-computationalism and its alignment - besides the mentioned differential nuances - with respect to modelling interpretation at the level of cognitive response (§ 3.3.2, though info-computationalism is actually proposed as to model throughout all levels of complexity). Not far from this approach, and covering what we have identified as reflexive and socio-ethical responses (§ 3.3.3-4), one of the authors has advanced a computational approach to the modelling of research processes in which not only deductive and inductive paths are focused, but particularly the fundamental role of creative abductions (Zimmermann and Wiedemann 2012).

Staying at the formal aspects, the categorical approach provided by Burgin in his General Theory of Information (GTI) offers an interesting toehold (Burgin 2012). Indeed, as we have upheld in the progressive perspective, the underlying structure of the world can well be modelled as to the general aspects of emergence through category theory (cf. Zimmermann and Díaz Nafría 2012, appendix 6). On the other hand, we have highlighted information as one fundamental ingredient for general agent dynamics (namely as potentiality for the selection of proper changes). Hence, the GTI seems to be valuable for the development of a UTI in both qualitative and quantitative senses, particularly considering the proven consistency with well-established theories of information. Nevertheless, recalling the aforementioned shyness with respect to formalisation within the UTI project, we also cherish the need to stress the modelling of information throughout nature in consistency with its related scientific knowledge. We consider such development should rely on the sciences, and requires proving with seemless consistency. Hence, a UTI should neither be a philosophia prima as Floridi defends, nor a mere formalising toolkit; rather a suitable philosphia ultima - as one of us has defended elsewhere (Zimmermann 2010), providing by those means an appropriate scaffold for the understanding of information in relation to other fundamental aspects of the world throughout the sciences - though neither reducing to them nor putting aside fundamental questioning.

Concerning the kernel question of the emergence of meaning, Brier's Cybersemiotics (2008; 2010) shows also some parallelism with our approach, which similarly develops an understanding of emergence in joint-venture with a general understanding of information. However, differently to Brier, we give a step forward so as to consider the foundation of meaning not only upon the "sign games" played by living beings, but also upon what might be named "spin games" played at the very world foundation - at pre-geometrical levels, as argued in the first part. Moreover, unlike in the cybersemiotic approach, we deem information to entail meaning in the first place - as we have discussed extensively - though we agree on the necessity of elaborating meanings of higher order through living, cognitive and social agency, as argued in the last section.

The terms of message and messenger as proposed by Capurro's Angeletics (2010; 2011) can be used to visualise the meaning-offer and the first-order interaction determined by a particular agency, as referred to in $\S 2$ (for instance the interaction which enables the emergence of the electromagnetic field). As far as the existence of the messenger constitutes a necessary condition for the message, in our scaffold, it is the first-order agency that is needed for the emergence of meaning in the first place - even at the most fundamental level. But insofar as the existence of an appropriate recipient enables the hermeneutical disclosing of meanings, it is the second-order agency (rooted on the same possibility than the first-order one) that enables the emergence of secondorder meanings and even the unveiling of the first-order ones. In similar terms, one of the authors has shown the complementarity of both programmes in a recent contribution (Díaz Nafría 2011).

Summarizing, our proposal contributes to the erection of a Unified Theory of Information according to a reviewed GDI which allows visualising information in nature altogether, complying with the 
scientific development and being able to collaborate with other approaches in order to achieve a better understanding of information, computation, meaning, interpretation and evolution of complexity.

As we argued at the beginning, it is the commonality of the way up and the way down what enables us to overcome the hindrances of a dualistic position so as to properly tackle the problem of understanding the emergence of meaning alongside the emergence of being throughout the hierarchy of complexity. The key-player in the advancement through the levels of complexity is the autonomous agent, through which emergence occurs in both ontological and epistemological senses: creating novelty in nature, and making that nature acknowledges itself. This onto-epistemic vision can be nicely symbolized by Herbert's verses concerning human's worldliness:

"His eyes dismount the highest star; $\mathrm{He}$ is in little all the sphere; Herbs gladly cure our flesh, because that they Find their acquaintance there." (George Herbert, 1633, Man, v.21-24)

\section{Appendix}

The problem of radiation, stated above, can easily be analysed by considering the Maxwell equations for a given frequency $\omega=2 \pi f$ :

$$
\begin{array}{ll}
\nabla \times \mathbf{E}=-\mathbf{M}-i \omega \boldsymbol{\mu} \mathbf{H} & \varepsilon \cdot \nabla \cdot \mathbf{E}=q_{e} \\
\nabla \times \mathbf{H}=\mathbf{J}+i \omega \varepsilon \mathbf{E} & \mu \cdot \nabla \cdot \mathbf{H}=q_{m}
\end{array}
$$

where $\mathbf{E}$ and $\mathbf{H}$ stand for the intensity distributions of the electric and magnetic field, respectively; $\mathbf{J}$ and $\mathbf{M}$ for electric and magnetic current densities; $q_{e}$ and $q_{m}$ for electric and magnetic charge distributions; and $\varepsilon$ and $\mu$ for the electric permittivity and magnetic permittivity of the medium.

The problem of relating the wave fields to the sources can be simplified through definition of the well-known vector potentials $\mathbf{A}$ and $\mathbf{F}$ :

$$
\begin{aligned}
& \mathbf{E}=-i \omega\left\{1+\frac{\nabla \nabla}{k^{2}}\right\} \mathbf{A}-\frac{1}{\varepsilon} \nabla \times \mathbf{F} \\
& \mathbf{H}=-i \omega\left\{1+\frac{\nabla \nabla}{k^{2}}\right\} \mathbf{F}+\frac{1}{\mu} \nabla \times \mathbf{A}
\end{aligned}
$$

which verify the wave equations directly related to the current distributions $\mathbf{J}$ and $\mathbf{M}$ - being $k$ the wave number, $k^{2}=\omega^{2} \varepsilon \mu$ :

$$
\begin{aligned}
& \nabla^{2} \mathbf{A}+k^{2} \mathbf{A}=-\mu \mathbf{J} \\
& \nabla^{2} \mathbf{F}+k^{2} \mathbf{F}=-\varepsilon \mathbf{M}
\end{aligned}
$$

Through (A3) the vector potentials can be described as linear superposition of Green distributions $G\left(\mathbf{r}, \mathbf{r}^{\prime}\right)=\frac{e^{-i k\left|\mathbf{r}-\mathbf{r}^{\prime}\right|}}{4 \pi\left|\mathbf{r}-\mathbf{r}^{\prime}\right|}$, where $\mathbf{r}^{\prime}$ represents the position of a punctual source, $\mathbf{r}$ the position in which the field is evaluated:

$$
\begin{aligned}
& \mathbf{A}(\mathbf{r})=\mu \iiint_{V} \mathbf{J}\left(\mathbf{r}^{\prime}\right) \cdot G\left(\mathbf{r}-\mathbf{r}^{\prime}\right) d v^{\prime}=\mu \mathbf{J}(\mathbf{r}) * G(\mathbf{r}) \\
& \mathbf{F}(\mathbf{r})=\varepsilon \iint_{V^{\prime}} \mathbf{M}\left(\mathbf{r}^{\prime}\right) \cdot G\left(\mathbf{r}-\mathbf{r}^{\prime}\right) d v^{\prime}=\varepsilon \mathbf{M}(\mathbf{r}) * G(\mathbf{r})
\end{aligned}
$$

being $V$ ' the volume of the source object, $*$ the tri-dimensional convolution. Using these vector potential definitions, the electric and magnetic field intensity distributions can also be derived, which can be described in terms of generalized Green tensors:

$$
\left[\begin{array}{l}
\mathbf{E}(\mathbf{r}) \\
\mathbf{H}(\mathbf{r})
\end{array}\right]=\left[\begin{array}{cc}
\mathbf{G}_{1}(\mathbf{r}) & \mathbf{G}_{2}(\mathbf{r}) \\
-\mathbf{G}_{2}(\mathbf{r}) & \frac{\varepsilon}{\mu} \mathbf{G}_{1}(\mathbf{r})
\end{array}\right] *\left[\begin{array}{l}
\mathbf{J}(\mathbf{r}) \\
\mathbf{M}(\mathbf{r})
\end{array}\right]=\mathbf{G}_{E, H}(\mathbf{r}) * \mathbf{S}(\mathbf{r})
$$

where $\mathbf{s}(\mathbf{r})$ denotes generalized sources (electric and magnetic). 
Since according to the uniqueness theorem, the field $\mathbf{E}, \mathbf{H}$ is unique when the superficial distribution of the either the tangent electric $\mathbf{E}$ or the magnetic $\mathbf{H}$ is specified on $S$, it is enough to focus on just one of the fields (or any combination of both), symbolized by $\boldsymbol{\Psi}(\mathbf{r})-$ as the phenomenon of the source-object: $\boldsymbol{\Psi}(\mathbf{r})=\mathbf{G}(\mathbf{r}) * \mathbf{s}(\mathbf{r})$.

\section{References}

Aguado, Juan. 2009. Information, Self-Reference and Observation Theory in the Context of Social Sciences Epistemology. triplec, 7(2): 344-356.

Ashtekar, Abhay. 1998. Geometric Issues in Quantum Gravity. In The Geometric Universe. Science, Geometry, and the Work of Roger Penrose, edited by Stephen Huggett, Lionel Mason, K. Paul Tod, Sheung Tsou, and Nicholas Woodhouse, 173-194. Oxford: Oxford University Press.

Balanis, Constantine. 1989. Advanced Engineering Electromagnetic. New York: John Wiley \& Sons.

Baez, John, ed. 1994. Knots and Quantum Gravity. Oxford: Clarendon

Barabási, Albert-László. 2002. Linked. The New Science of Networks. Cambridge: Perseus.

Barbieri, Marcello 2003. The Organic Codes: An Introduction to Semantic Biology. Cambridge: Cambridge University Press.

Barrow, John, Paul Davies, and Charles Harper Jr., eds. 2004. Science and Ultimate Reality: Quantum Theory, Cosmology, and Complexity. Cambridge: Cambridge University Press.

Benenti, Guiliano, Guilio Casati, and Giuliano Strini. 2004 (2007). Principles of Quantum Computation and Information. (2 vols.) Singapore: World Scientific.

Berman, Gennady, Gary Doolen, Ronnie Mainieri, and Vladimir Tsifrinovich. 1998. Introduction to Quantum Computers. Singapore: World Scientific.

Brier, Søren. 2008. Cybersemiotics: Why Information is not Enough! Toronto: University of Toronto Press.

Brier, Søren. 2011. Cybersemiotics. In GlossariumBITri: Glossary of Concepts, Metaphors, Theories and Problems Concerning Information, edited by José María Díaz Nafría, Mario Pérez-Montoro, and Francisco Salto. León: Universidad de León.

Bub, Jeffrey. 1997. Interpreting the Quantum World. Cambridge: Cambridge University Press.

Burgin, Mark. 2010. Theory of Information: Fundamentality, Diversity and Unification. Singapore: World Scientific Publishing.

Burgin, Mark, and José María Díaz Nafría. 2011. Algorithmic Information Theory In GlossariumBITri: Glossary of Concepts, Metaphors, Theories and Problems concerning Information, edited by José María Díaz Nafría, Mario Pérez-Montoro, and Francisco Salto. León: Universidad de León.

Bruner, Jerome, and Cecile Goodman. 1947. Value and Need as Organizing Factors in Perception. Journal of Abnormal and Social Psychology, 42: 33-44.

Capurro, Raphael. 2011. Angeletics. In GlossariumBITri: Glossary of Concepts, Metaphors, Theories and Problems Concerning Information, edited by José María Díaz Nafría, Mario Pérez-Montoro, and Francisco Salto. León: Universidad de León.

Capurro, Raphael, and John Holgate, eds. 2011. Messages and Messengers. Munich: Wilhelm Fink.

Capurro, Raphael, Peter Fleissner, and Wolfgang Hofkirchner. 1999. Is a Unified Theory of Information Feasible? A Trialogue. (e-mail discussion). In The Quest for a Unified Theory of Information, edited by Wolfgang Hofkirchner, 9-30. Amsterdam: Gordon \& Breach.

Copeland, B. Jack. 2004. Computation. In Philosophy of Computing and Information, edited by Luciano Floridi, 3-17. Oxford: Blackwell.

Crutchfield, James. 1994. The Calculi of Emergence. Santa Fe Institute working paper SFI 94-03-016, (Physica D special issue: Proceedings of the Oji International Seminar on Complex Systems.)

Curcio, Christine, Kenneth Sloan, Orin Packer, Anita Hendrickson, and Robert Kalina. 1987. Distribution of Cones in Human and Monkey Retina: Individual Variability and Radial Asymmetry. Science, 236: 579-582.

Davies, Paul 2004. John Archibald Wheeler and the clash of ideas. In Science and Ultimate Reality. Quantum Theory, Cosmology, and Complexity, edited by John Barrow, Paul Davies, and Charles Harper Jr., 3-23. Cambridge: Cambridge University Press.

Deutsch, David. 2004a. Qubit Field Theory. arXiv:quant-ph/0401024. Accessed July 19, 2011: http://arxiv.org/ftp/quantph/papers/0401/0401024.pdf

Deutsch, David. 2004b. It from qubit. In Science and Ultimate Reality. Quantum Theory, Cosmology, and Complexity, edited by John Barrow, Paul Davies, and Charles Harper Jr., 90-102. Cambridge: Cambridge University Press.

Díaz Nafría, José María. 2003. Contribución en métodos inversos para la caracterización de sistemas radiantes (Contributions in inverse methods for the characterization of radiant systems). Madrid: Universidad Politécnica de Madrid. Accessed January 30, 2011: http://www.novatores.org/html/es/eprint/show.html?ePrintld=119

Díaz Nafría, José María. 2008. La inderminación de la observación [The indeterminacy of observation]. In What is information?, edited by José María Díaz Nafría and Francisco Salto. León: Publicaciones de la Universidad de León. Accessed December 23,2011: http://bitrum.wordpress.com/2009/06/07/397/

Díaz Nafría, José María. 2011. Messages in an Open Universe. In Messages and Messengers, edited by R. Capurro and J. Holgate, 195-228. Munich: Wilhelm Fink.

Díaz Nafría, José María, and Mario Pérez-Montoro. 2011a. Is Information a Sufficient Basis for Cognition? (Part 1: Critique on Dretske's vision). tripleC, 9 (2): 358-366.

Díaz Nafría, José María, and Mario Pérez-Montoro. 2011b. Is Information a Sufficient Basis for Cognition? (Part 2: Physical Foundations). tripleC, 9 (2): 367-376.

Díaz Nafría, José María, Mario Pérez-Montoro, and Francisco Salto, eds. GlossariumBITri: Glossary of Concepts, Metaphors, Theories and Problems Concerning Information. León: Universidad de León, 2011. Accessed December 15, 2011: http://glossarium.bitrum.unileon.es/glossary/

Díaz Nafría, José María, and Franciso Salto, eds. 2009, What is really information? Special Issue: tripleC 7(2). Accessed December 15, 2011: http://bitrumcontributions.files.wordpress.com/2012/02/what-is-really-information.pdf 
Díaz Nafría, José María, and Rainer Zimmermann. 2012. Emergence and Evolution of Meaning. The GDI Revisiting Programme. Part II: The Regressive Perspective: Bottom-Up. To be published. Draft accessed June 15, 2012: http://bitrumcontributions.wordpress.com/

Dodig-Crnkovic, Gordana. 2011. Info-Computationalism. In GlossariumBITri: Glossary of Concepts, Metaphors, Theories and Problems concerning Information, edited by José María Díaz Nafría, Mario Pérez-Montoro, and Francisco Salto. León: Universidad de León.

Fenzl, Norbert, and Wolfgang Hofkirchner. 1997. Information Processing in Evolutionary Systems. In Schweitzer, F. (Ed.), Self-Organisation of Complex Structures: From Individual to Collective Dynamics, 59-70. London: Gordon and Breach.

Floridi, Luciano, ed. 2004. Philosophy of Computing and Information. Oxford: Blackwell.

Floridi, Luciano. 2004. Information. In Philosophy of Computing and Information, edited by Luciano Floridi, 40-61. Oxford: Blackwell.

Floridi, Luciano. 2010a. Semantic Information and the Network Theory of Account. Synthesis 2010, DOI: 10.1007/s11229010-9821-4.

Floridi, Luciano. 2010b. Information, Possible Worlds, and the Cooptation of Scepticism. Synthese, 175: 63-88.

Floridi, Luciano. 2011a. A Defence of Constructionism: Philosophy as Conceptual Engineering. Metaphilosophy, 42: 282304.

Floridi, Luciano. 2011b. The Philosophy of Information. Oxford: Oxford University Press.

Foerster, Heinz von. 1981. Observing Systems. Seaside: Intersystems Publications.

Gambini, Rodolfo, and Jorge Pullin. 1996. Loops, Knots, Gauge Theories and Quantum Gravity. Cambridge: Cambridge University Press.

Gray, Henry. 1918. Anatomy of the Human Body. Philadelphia: Lea \& Febiger.

Haefner, Klaus. 1999. Foreword. In The Quest for a Unified Theory of Information, edited by Wolfgang Hofkirchner, xiii-xvii. Amsterdam: Gordon \& Breach

Halliwell, Jonathan, Juan Pérez-Mercader, and Wojciech Zurek, eds. 1994. Physical Origin of Time Asymmetry. Cambridge: Cambridge University Press.

Hawking, Stephen, and Roger Penrose. 1996. The Nature of Space and Time. Princeton: Princeton University Press.

Hoffmeyer, Jesper. 2010. Semiotic Freedom: An Emerging Force. In Information and the Nature of Reality: From Physics to Metaphysics, edited by Paul Davies and Niels Gregersen, 185-204. Cambridge: Cambridge University Press.

Hofkirchner, Wolfgang, ed. 1999. The Quest for a Unified Theory of Information. Amsterdam: Gordon \& Breach.

Hofkirchner, Wolfgang. 2010. Twenty Questions About a Unified Theory of Information. Litchfield Park: Emergent Publications.

Hofkirchner, Wolfgang. 2012. Emergent Information: An Outline Unified Theory of Information Framework. Singapore: World Scientific Publishing.

Huggett, Stephen, Lionel Mason, K. Paul Tod, Sheung Tsou, and Nicholas Woodhouse, eds. 1998. The Geometric Universe. Science, Geometry, and the Work of Roger Penrose. Oxford: Oxford University Press.

Jantsch, Erich. 1982. Die Selbstorganisation des Universums. [Self-Organization of the Universe]. Muenchen: dtv Wissenschaft (Muenchen, Germany: Hanser, 1979).

Johnson, S. 2002. Emergence. London: Penguin.

Kauffman, Louis. 1998. Spin Networks and Topology. In The Geometric Universe. Science, Geometry, and the Work of Roger Penrose, edited by Stephen Huggett, Lionel Mason, K. Paul Tod, Sheung Tsou, and Nicholas Woodhouse, 277289. Oxford: Oxford University Press.

Kauffman, Stuart. 2000. Investigations. Oxford: Oxford University Press.

Kauffman, Stuart. 2004. Autonomous agents. In Science and Ultimate Reality. Quantum Theory, Cosmology, and Complexity, edited by John Barrow, Paul Davies, and Charles Harper Jr., 654-666. Cambridge: Cambridge University Press.

Kauffman, Stuart, and Philip Clayton. 2006. On Emergence, Agency, and Organization. Biology and Philosophy, 21: 501521.

Lamb, Trevor D. 2011. Evolution of the Eye. Scientific American, 305 (1): July, 48-53.

Lawvere, F. William, and Robert Rosebrugh. 2003. Sets for Mathematics. Cambridge: Cambridge University Press.

Levi-Strauss, C. 1967. The Story of Asdiwal. In The Structural Study of Myth and Totetism edited by Edmund Leach, 1-48. London: Routledge.

Levi-Strauss, Claude. 1969 (orig. 1949). The Elementary Structures of Kinship, Oxford: Alden Press.

Lloyd, Seth. 2006. Programming the Universe. New York: Vintage (Random House).

Lloyd, Seth. 2010. The Computational Universe. In Information and the Nature of Reality: From Physics to Metaphysics, edited by Paul Davies and Niels Gregersen, 92-103. Cambridge: Cambridge University Press.

Magnon, Anne. 1997. Arrow of Time and Reality. Singapore: World Scientific.

Mainzer, Klaus. 2004. An Introduction to Systems Science. In Philosophy of Computing and Information, edited by Luciano Floridi, 28-39. Oxford, UK: Blackwell.

McMullin, Ernan. 2010. From Matter to Materialism ... and (almost) back. In Information and the Nature of Reality: From Physics to Metaphysics, edited by Paul Davies and Niels Gregersen, 13-37. Cambridge: Cambridge University Press.

Ogura Atsushi, Kazuho Ikeo, and Takashi Gojobori. 2004. Comparative Analysis of Gene Expression for Convergent Evolution of Camera Eye Between Octopus and Human. Genome Res., 14: 1555-1561. Accessed December 20, 2011: http://www.genome.org/cgi/doi/10.1101/gr.2268104

PEET (2010). The Euglenoid Project (web). Partnership for Enhancing Partnership in Taxonomy-National Science Foundation. Accessed July $2010 \mathrm{http://euglena.msu.edu}$

Penrose, Roger. 1989. The Emperor's New Mind. Oxford: Oxford University Press.

Penrose, Roger. 1994. Shadows of the Mind. Oxford: Oxford University Press.

Penrose, Roger. 1997. The Large, the Small, and the Human Mind. Cambridge: Cambridge University Press.

Penrose, Roger. 2005. The Road to Reality: A Complete Guide to the Laws of the Universe. New York: Knopf.

Prigogine, llya. 1979. From Being to Becoming. [German edition] Muenchen: Piper.

Prigogine, llya. 1996. The End of Certainty. New York: Free Press.

Raisbeck, Alden M.D. 1954. Nervous System. In The American Peoples Encyclopedia. Chicago, Volume 14, 466. 
Rovelli, Carlo. 2004. Quantum Gravity. Cambridge: Cambridge University Press.

Schelkunoff, Sergei 1936. Some Equivalence Theorems of Electromagnetics and their Application to Radiation Problems. Bell System Tech. J., 15: 92-112.

Smolin, Lee. 1997. The Life of the Cosmos. Oxford: Oxford University Press.

Smolin, Lee. 2000. Three Roads to Quantum Gravity. London: Weidenfeld \& Nicolson.

Smolin, Lee. 2004. Quantum Theories of Gravity: Rresults and Prospects. In Science and Ultimate Reality. Quantum Theory, Cosmology, and Complexity, edited by John Barrow, Paul Davies, and Charles Harper Jr., 492-527. Cambridge: Cambridge University Press.

Smolin, Lee. 2006. The Trouble with Physics. New York: Houghton Mifflin.

Sobrino, José, and José Simón. 1986. Neurofisiología. Madrid: Luzan 5.

Spinoza, Baruch. 2003 (orig. 1677). Ethics. Gutenberg Project. Accessed October 10, 2011: http://www.gutenberg.org/ebooks/3800

Susskind, Leonard, and James Lindesay. 2005. The Holographic Universe: An Introduction to Black Holes, Information, and the String Theory Revolution. Singapore: World Scientific.

Taborsky, Edwina, ed. 1999. Semiosis, Evolution, Energy. Towards a Reconceptualization of the Sign. Aachen: Shaker.

Thom, René. 1973. Langage et catastrophes. Elements pour une sémantique topologique. [Elements of a Topological Semantics.] In Peixoto M.M. ed. Dynamical Systems, 619-654. Waltham, Mass.: Academic Press.

Thom, René. 1975. Structural Stability and Morphogenesis. Reading: Benjamin.

Thom, René. 1983. Mathematical Models of Morphogenesis. Chichester: Horwood/Wiley.

Urquhart, Alasdair. 2004. Complexity. In Philosophy of Computing and Information, edited by Luciano Floridi, 18-27. Oxford: Blackwell.

Vedral, Vlatko. 2010. Decoding Reality. Oxford: Oxford University Press.

Yetter, David. 2001. Functorial Knot Theory: Categories and Tangles, Coherence, Categorical Deformations, and Topological Invariants. Singapore: World Scientific.

Young, John. 1962. The Retina of Cephalopods and Its Degeneration After Optic Nerve Section. Phil. Trans. R. Soc. Lond. $B, 245$ (718): 1-18

Weizsaecker, Carl von. 1971. Die Einheit der Natur. [Unity of Nature] Muenchen: Hanser.

Zimmermann, Rainer. 1974. Limits of Research in Modern Physics? J. Kingsway (London), 10 (65): 25-29.

Zimmermann, Rainer. 1978. The Morphogenesis of Black Holes. Berlin: FU Berlin.

Zimmermann, Rainer. 1982. The Lepton Brusselator. Creation of Structure in the Early Universe. J. GRG, 14 (11): 10511060 .

Zimmermann, Rainer. 1982. Homogeneous Cosmologies and their Stability Behaviour. New Physics (Korean Physical Society) 22 (3): 291-315.

Zimmermann, Rainer. 1984. Non-Equilibrium Thermodynamics of Cosmological Event Horizons. ZAMM 64(4/5), T402T404.

Zimmermann, Rainer. 1990. Twistors and Self-Reference: On the Generic Non-Linearity of Nature. Philosophia Naturalis, 27 (2): 272-297.

Zimmermann, Rainer. 1991. Selbstrefrenz und poetische Praxis [Self-Reference and poetical praxis]. Cuxhaven: Junghans.

Zimmermann, Rainer. 1998a. Die Rekonstruktion von Raum, Zeit und Materie [The Reconstruction of Space, Time, and Matter = On Schelling's Philosophy of Nature]. Frankfurt am Main: Peter Lang.

Zimmermann, Rainer. 2000a. Loops and Knots as Topoi of Substance. Spinoza Revisited. arXiv:gr-qc/0004077, 41. Accessed July 28, 2011: www.arxiv.org/pdf/gr-qc/0004077

Zimmermann, Rainer 2001. René Thom - Semiologie des Chaos [Semiology of Chaos]. In Franzoesische Nachkriegsphilosophie [French Post-War Philosophy], edited by Günter Abel, 185-203. Berlin: Nomos.

Zimmermann, Rainer. 2002. Spinoza in Context: A Holistic Approach in Modern Terms. In Infinity, Causality, and Determinism. Cosmological Enterprises and their Preconditions, edited by Eeva Martikainen,165-186). Frankfurt am Main: Peter Lang.

Zimmermann, Rainer. 2004a. Otherland Revisited. Philosophical Implications of Artificial Worlds. Part 1: City of Golden Shadow or the Ontology of Artificial Worlds. In Science of Self-Organization and Self-Organization of Science (INTAS Volume of Collected Essays 2), edited by Irina Dobronravova and Wolfgang Hofkirchner, 86-116. Kyiv: Abris.

Zimmermann, Rainer. 2004b. System des transzendentalen Materialismus. [System of Transcendental Materialism] Paderborn: Mentis.

Zimmermann, Rainer. 2005. Otherland Revisited: Philosophical Implications of Artificial Worlds. Part 2: River of Blue Fire or the Epistemology of Artificial Worlds. In Towards Otherland, Languages of Science and Languages Beyond, edited by Rainer Zimmermann and Vladimir Budanov, 29-44, (INTAS Volume of Collected Essays 3). Kassel: Kassel University Press.

Zimmermann, Rainer. 2006. “... the exact size, shape and color of hope itself." Virtual Environment \& Concrete Utopia. In Heimat in vernetzten Welten, edited by Francesca Vidal, 67-82. Moessingen: Talheimer.

Zimmermann, Rainer. 2007a. Topological Aspects of Biosemiotics. tripleC, 5 (2, special issue): 49-63.

Zimmermann, Rainer. 2007b. On the Modality of the World: Space and Time in Spinoza. In Notions of Space and Time, edited by Frank Linhard and Peter Eisenhardt, 217-242. Frankfurt am Main: Klostermann.

Zimmermann, Rainer. 2010. New Ethics Proved in Geometrical Order. Spinozist Reflexions on Evolutionary Systems. Litchfield Park: Emergent Publications.

Zimmermann, Rainer. 2011. Basic Concepts of Transcendental Materialism. Invited Lecture at the Kingston University, London, February 2011. Accessed July 28, 2011: http://backdoorbroadcasting.net/2011/02/rainer-e-zimmermann-basicconcepts-of-transcendental-materialism/

Zimmermann, Rainer, and José María Díaz Nafría. 2012. Emergence and Evolution of Meaning: The GDI Revisiting Programme. Part I: The Progressive Perspective: Top-Down. In Information, in press. Draft accessed December 15, 2011 : http://bitrumcontributions.wordpress.com/ 
Zimmermann, Rainer, and Anna Soci. 2004. The Emergence of Bologna and its Future Consequences. Decentralization as Cohesion Catalyst in Guild-Dominated Urban Networks. In The Information Society. Understanding its Institutions Interdisciplinary 181-182. Maastricht: EAEPE. Accessed July 31, 2011: www.arxiv.org/pdf/cond-mat/0411509

Zimmermann, Rainer, and Simon Wiedemann. 2012. Kreativität und Form: Programm eines Glasperlenspiels zum Experimentieren mit Wissen (Creativity and Form: Programme of a Glass Bead Game for Experimenting with knowledge). Berlin: Springer Verlag.

\section{About the Authors}

\section{Rainer E. Zimmerman}

received his PhD in Mathematics in 1977 from FU Berlin) and spent the years between 1982-1988 in Studies in Philosophy, History, and Literature at TU Berlin. He received a PhD in Philosophy in 1988 from TU Berlin. He has held the following positions: since 1995 Professor of Philosophy at the Polytechnic University of Muenchen; In 1998, Habilitation in Natural Philosophy (University of Kassel) - since then member (Privatdozent) of the department there; 1999/2000 Visiting Scholar at the History and Philosophy of Science Department and Visiting Fellow of Clare Hall, both at Cambridge (UK) - since then Life Member of Clare Hall; 2001 Research Visitor to the University of Bologna, Cooperative Research Project there under the title "Reconstruction of the Historical City Centre"; 2003 Senior Visiting Fellow to the Institute of Advanced Studies, Villa Gandolfi Pallavicini, University of Bologna; 2006 International Visiting Professor at the Information and Communication Technologies and Society program, University of Salzburg; 2010/2011 Visiting Professor, Centre for Metropolitan Studies, TU Berlin.

\section{J.M. Díaz Nafría}

obtained M.Sc. in telecommunication engineering from the Universidad del País Vasco, Bilbao, Spain, and received his PhD in telecommunication engineering from the Universidad Politécnica de Madrid with a dissertation on "Contributions to the electromagnetic inverse problem". He was also awarded with a M.Sc. in Philosophy by the Universidad Nacional de Educación a Distancia (UNED). He is currently researcher at the Universidad of León, visiting professor at the Munich University of Applied Sciences, and belongs to the board of directors of the Science of Information Institute, the Institute für Design Science, and the International Society of Information Studies. He is also member of several international scientific societies in the field of information theories. He was research fellow at the Vienna University of Technology and at the Technical University of Madrid. He also served as professor at the Universidad Alfonso X el Sabio in Madrid between 1997 and 2009 and has been visiting lecturer at the University of Furtwangen, Sankt Pölten University of Applied Sciences and University of Salzburg. He co-directed the "First International Meeting of Experts in Information Theories" (León, Spain, 2008) and the "Colloquium BITae" (León, Spain, 2009). He currently coordinates an interdisciplinary research group meted around the BITrum project (Interdisciplinary approach to information, http://en.bitrum.unileon.es). 